\title{
Expert consensus on resection of chest wall tumors and chest
}

\section{wall reconstruction}

\author{
Lei Wang ${ }^{1 \#}$, Xiaolong Yan ${ }^{1 \#}$, Jinbo Zhao ${ }^{1 \#}$, Chang Chen ${ }^{2}$, Chun Chen ${ }^{3}$, Jun Chen ${ }^{4}, \mathrm{Ke}-\mathrm{Neng} \mathrm{Chen}^{5}$, \\ Tiesheng $\mathrm{Cao}^{6}$, Ming-Wu Chen ${ }^{7}$, Hongbin Duan ${ }^{8}$, Junqiang Fan ${ }^{9}$, Junke Fu ${ }^{10}$, Shugeng Gao ${ }^{11}$, \\ Hui Guo ${ }^{12}$, Shiping Guo ${ }^{13}$, Wei Guo ${ }^{14}$, Yongtao Han ${ }^{15}$, Ge-Ning Jiang ${ }^{2}$, Hongjing Jiang ${ }^{16}$, Wen-Jie Jiao ${ }^{17}$, \\ Mingqiang Kang ${ }^{3}$, Xuefeng Leng ${ }^{15}$, He-Cheng $\mathrm{Li}^{18}$, Jing Li ${ }^{19}$, Jian $\mathrm{Li}^{20}$, Shao-Min Li ${ }^{21}$, Shuben $\mathrm{Li}^{22}$, \\ Zhigang $\mathrm{Li}^{23}$, Zhongcheng $\mathrm{Li}^{24}$, Chaoyang Liang ${ }^{25}$, Nai-Quan $\mathrm{Mao}^{26}$, Hong $\mathrm{Mei}^{27}$, Daqiang Sun ${ }^{28}$, \\ Dong Wang ${ }^{29}$, Luming Wang ${ }^{30}$, Qun Wang ${ }^{31}$, Shumin Wang ${ }^{32}$, Tianhu Wang ${ }^{33}$, Lunxu Liu ${ }^{34}$, \\ Gaoming Xiao ${ }^{35}$, Shidong $\mathrm{Xu}^{36}$, Jinliang Yang ${ }^{37}$, Ting $\mathrm{Ye}^{38}$, Guangjian Zhang ${ }^{10}$, Linyou Zhang ${ }^{39}$, \\ Guofang Zhao ${ }^{40}$, Jun Zhao ${ }^{11}$, Wen-Zhao Zhong ${ }^{41}$, Yuming Zhu ${ }^{2}$, Karel W. E. Hulsewéé ${ }^{42}$, \\ Yvonne L. J. Vissers ${ }^{42}$, Erik R. de Loos ${ }^{42}$, Jin Yong Jeong ${ }^{43}$, Giuseppe Marulli ${ }^{44}$, Alberto Sandri ${ }^{45}$, \\ Zsolt Sziklavari $^{46,47}$, Jacopo Vannucci ${ }^{48}$, Luca Ampollini ${ }^{49}$, Yuichiro Ueda ${ }^{50}$, Chaozong Liu ${ }^{51}$, Andrea Bille ${ }^{52}$, \\ Masatsugu Hamaji ${ }^{53}$, Beatrice Aramini ${ }^{54}$, Ilhan Inci ${ }^{55}$, Cecilia Pompili ${ }^{56}$, Hans Van Veer ${ }^{57}$, \\ Alfonso Fiorelli ${ }^{58}$, Ricciardi Sara ${ }^{59}$, Inderpal S. Sarkaria ${ }^{60}$, Fabio Davoli ${ }^{61}$, Hiroaki Kuroda ${ }^{62}$, \\ Servet Bölükbas ${ }^{63}$, Xiao-Fei Li ${ }^{1}$, Lijun Huang ${ }^{1}$, Tao Jiang ${ }^{1}$
}

${ }^{1}$ Department of Thoracic Surgery, Tangdu Hospital, Air Force Medical University, Xi'an, China; ${ }^{2}$ Department of Thoracic Surgery, Shanghai Pulmonary Hospital, Tongji University School of Medicine, Shanghai, China; ${ }^{3}$ Department of Thoracic Surgery, Fujian Medical University Union Hospital, Fuzhou, China; ${ }^{4}$ Tianjin Key Laboratory of Lung Cancer Metastasis and Tumor Microenvironment, Lung Cancer Institute, Tianjin Medical University General Hospital, Tianjin, China; ${ }^{5}$ Department of Thoracic Surgery, Key Laboratory of Carcinogenesis and Translational Research (Ministry of Education), Peking University Cancer Hospital \& Institute, Beijing, China; ${ }^{6}$ Department of Ultrasound Diagnosis, Tangdu Hospital, Air Force Medical University, Xi'an, China; ${ }^{7}$ Department of Cardiothoracic Surgery, The First Affiliated Hospital of Guangxi Medical University, Nanning, China; ${ }^{8}$ Department of Thoracic Surgery, Zhongshan Hospital Affiliated to Xiamen University, Xiamen, China; ${ }^{9}$ Department of Thoracic Surgery, Second Affiliated Hospital of Zhejiang University, School of Medicine, Hangzhou, China; ${ }^{10}$ Department of Thoracic Surgery, First Affiliated Hospital of Xi'an Jiaotong University, Xi'an, China; ${ }^{11}$ Department of Thoracic Surgery, National Cancer Center/National Clinical Research Center for Cancer/Cancer Hospital, Chinese Academy of Medical Sciences and Peking Union Medical College, Beijing, China; ${ }^{12}$ Department of Medical Oncology, First Affiliated Hospital of Xi'an Jiaotong University, Xi'an, China; ${ }^{13}$ Department of Thoracic Surgery, The Tumor Hospital Affiliated to Shanxi Medical University, Taiyuan, China; ${ }^{14}$ Department of Thoracic Surgery, Daping Hospital, Army Medical University, Chongqing, China; ${ }^{15}$ Department of Thoracic Surgery, Sichuan Cancer Hospital \& Research Institute, School of Medicine, University of Electronic Science and Technology of China (UESTC), Chengdu, China; ${ }^{16}$ Department of Minimally Invasive Esophageal Surgery, Key Laboratory of Cancer Prevention and Therapy, National Clinical Research Center for Cancer, Tianjin Medical University Cancer Institute and Hospital, Tianjin, China; ${ }^{17}$ Department of Thoracic Surgery, The Affiliated Hospital of Qingdao University, Qingdao, China; ${ }^{18}$ Department of Thoracic Surgery, Ruijin Hospital, Shanghai Jiao Tong University School of Medicine, Shanghai, China; ${ }^{19}$ Department of Plastic and Burn Surgery, Tangdu Hospital, Air Force Medical University, Xi'an, China; ${ }^{20}$ Department of Thoracic Surgery, Peking University Third Hospital, Beijing, China; ${ }^{21}$ Department of Thoracic Surgery, the Second Affiliated Hospital of Xi'an Jiaotong University, Xi'an, China; ${ }^{22}$ Department of Thoracic Surgery, The First Affiliated Hospital of Guangzhou Medical University, Guangzhou, China; ${ }^{23}$ Department of Thoracic Surgery, Section of Esophageal Surgery, Shanghai Chest Hospital, Shanghai Jiao Tong University, Shanghai, China; ${ }^{24}$ Department of Thoracic and Cadiovascular Surgery, Affiliated Hospital of Qinghai University, Xining, China; ${ }^{25}$ Department of Thoracic Surgery, China-Japan Friendship Hospital, Beijing, China; ${ }^{26}$ Department of Thoracic Surgery, Tumor Hospital Affiliated to Guangxi Medical University, Nanning, China; ${ }^{27}$ Department of Thoracic Surgery, Guizhou Provincial People's Hospital, Guiyang, China; ${ }^{28}$ Department of Thoracic Surgery, Tianjin Chest Hospital, Tianjin, China; ${ }^{29}$ Department of Cardiothoracic Surgery, Affiliated Taikang Xianlin Drum Tower Hospital, Medical School of Nanjing University, Nanjing, China; ${ }^{30}$ Department of Thoracic Surgery, The First Affiliated Hospital, Zhejiang University School of Medicine, Hangzhou, China; ${ }^{31}$ Department of Thoracic Surgery, Zhongshan Hospital, Fudan University, Shanghai, China; ${ }^{32}$ Department of Thoracic Surgery, General Hospital of Shenyang Military Area, Shenyang, China; ${ }^{33}$ Department of Thoracic Surgery, The Third Affiliated Hospital of Chongqing Medical University, Chongqing, China; 
${ }^{34}$ Department of Thoracic Surgery, West China Hospital, Sichuan University, Chengdu, China; ${ }^{35}$ Department of Thoracic Surgery, Hunan Cancer Hospital and the Affiliated Cancer Hospital of Xiangya School of Medicine, Central South University, Changsha, China; ${ }^{36}$ Department of Thoracic Surgery and Oncology, Harbin Medical University Cancer Hospital, Harbin, China; ${ }^{37}$ Department of Thoracic Surgery, The Third Hospital of Hebei Medical University, Shijiazhuang, China; ${ }^{38}$ Department of Thoracic Surgery, Shanghai Cancer Center, Fudan University, Shanghai, China; ${ }^{39}$ Department of Thoracic Surgery, The Second Affiliated Hospital of Harbin Medical University, Harbin, China; ${ }^{40}$ Department of Cardiothoracic Surgery, Hwa Mei Hospital (Ningbo No. 2 Hospital), University of Chinese Academy of Sciences, Ningbo, China; ${ }^{41}$ Guangdong Provincial Key Laboratory of Translational Medicine in Lung Cancer, Guangdong Lung Cancer Institute, Guangdong Provincial People's Hospital and Guangdong Academy of Medical Sciences, Guangzhou, China; ${ }^{42}$ Division of General Thoracic Surgery, Department of Surgery, Zuyderland Medical Center, Heerlen, The Netherlands; ${ }^{43}$ Department of Thoracic and Cardiovascular Surgery, College of Medicine, The Catholic University of Korea, Seoul, Korea; ${ }^{44}$ Department of Emergency and Organ Transplantation, Thoracic Surgery Unit, University Hospital of Bari, Bari, Italy; ${ }^{45}$ Thoracic Surgery Division, Department of Oncology, San Luigi Gonzaga Hospital, Orbassano (To), Italy; ${ }^{46}$ Department of Thoracic Surgery, Klinikum Coburg, Coburg, Germany; ${ }^{47}$ Department of Thoracic Surgery, University Medical Center Regensburg, Regensburg, Germany; ${ }^{48}$ Department of Thoracic Surgery, University of Rome Sapienza, Policlinico Umberto I, Rome, Italy; ${ }^{49}$ Thoracic Surgery, Department of Medicine and Surgery, University Hospital of Parma, Parma, Italy; ${ }^{50}$ Department of General Thoracic, Breast and Pediatric Surgery, School of Medicine, Fukuoka University, Fukuoka, Japan; ${ }^{51}$ Institute of Orthopaedic \& Musculoskeletal Science, Division of Surgery \& Interventional Science, University College London, Royal National Orthopaedic Hospital, London, UK; ${ }^{52}$ Department of Thoracic Surgery, Guy's Hospital, London, UK; ${ }^{53}$ Department of Thoracic Surgery, Kyoto University Hospital, Kyoto, Japan; ${ }^{54}$ Division of Thoracic Surgery, Department of Medical and Surgical Sciences, University of Modena and Reggio Emilia, Modena, Italy; ${ }^{55}$ Department of Thoracic Surgery, University Hospital, Raemistrasse, Zurich, Switzerland; ${ }^{56}$ Department of Thoracic Surgery, AUOI Verona, Verona, Italy; ${ }^{57}$ Department of Thoracic Surgery, University Hospitals Leuven, Leuven, Belgium; ${ }^{58}$ Thoracic Surgery Unit, University of Campania Luigi Vanvitelli, Naples, Italy; ${ }^{59}$ Division of Thoracic Surgery, San Camillo Forlanini Hospital, Rome, Italy; ${ }^{60}$ Department of Thoracic Surgery, University of Pittsburgh Medical Center, Pittsburgh, PA, USA; ${ }^{61}$ Department of Thoracic Surgery, AUSL Romagna, S. Maria delle Croci Teaching Hospital, Ravenna, Italy; ${ }^{62}$ Department of Thoracic Surgery, Aichi Cancer Center Hospital, Nagoya, Japan; ${ }^{63}$ Department of Thoracic Surgery, Evang. Kliniken Essen-Mitte, Essen, Germany

\#These authors contributed equally to this work.

Correspondence to: Tao Jiang, MD; Lijun Huang, MD; Xiao-Fei Li, MD. Department of Thoracic Surgery, Tangdu Hospital, Air Force Medical University, Xi'an 710038, China. Email: jiangtaochest@163.com; hljyxq@fmmu.edu.cn; lxfchest@fmmu.edu.cn.

Abstract: Chest wall tumors are a relatively uncommon disease in clinical practice. Most of the published studies about chest wall tumors are usually single-center retrospective studies, involving few patients. Therefore, evidences regarding clinical conclusions about chest wall tumors are lacking, and some controversial issues have still to be agreed upon. In January 2019, 73 experts in thoracic surgery, plastic surgery, science, and engineering jointly released the Chinese Expert Consensus on Chest Wall Tumor Resection and Chest Wall Reconstruction (2018 edition). After that, numerous experts put forward new perspectives on some academic issues in this version of the consensus, pointing out the necessity to further discuss the points of contention. Thus, we conducted a survey through the administration of a questionnaire among 85 experts in the world. Consensus has been reached on some major points as follows. (I) Wide excision should be performed for desmoid tumor (DT) of chest wall. After excluding the distant metastasis by multi-disciplinary team, solitary sternal plasmacytoma can be treated with extensive resection and adjuvant radiotherapy. (II) Wide excision with above $2 \mathrm{~cm}$ margin distance should be attempted to obtain R0 resection margin for chest wall tumor unless the tumor involves vital organs or structures, including the great vessels, heart, trachea, joints, and spine. (III) For patients with chest wall tumors undergoing unplanned excision (UE) for the first time, it is necessary to carry out wide excision as soon as possible within 1-3 months following the previous surgery. (IV) Current Tumor Node Metastasis staging criteria (American Joint Committee on Cancer) of bone tumor and soft tissue sarcoma are not suitable for chest wall sarcomas. (V) It is necessary to use rigid implants for chest wall reconstruction once the maximum diameter of the chest wall defect exceeds $5 \mathrm{~cm}$ in adults and adolescents. (VI) For non-small cell lung cancer (NSCLC) invading the chest wall, wide excision with neoadjuvant and/or adjuvant therapy are recommended for patients with stage $T_{3-4} N_{0-1} M_{0}$. As clear guidelines are lacking, these consensus statements on controversial issues 
on chest wall tumors and resection could possibly serve as further guidance in clinical practice during the upcoming years.

Keywords: Chest wall tumor; excision margin; radiotherapy; chest wall reconstruction

Submitted Sep 18, 2021. Accepted for publication Nov 26, 2021.

doi: $10.21037 /$ tlcr-21-935

View this article at: https://dx.doi.org/10.21037/tlcr-21-935

\section{Introduction}

Chest wall tumors concern benign and malignant neoplasms involving the chest wall, including primary tumors arising from muscle, fat blood vessel, nerve sheath, cartilage, or bone, metastatic tumors, and local invasion of tumors arising from adjacent organs like the lung, mediastinum, pleura, or breast (1). The incidence of primary chest wall tumors is low, accounting for only $5 \%$ of all chest wall tumors. Most primary chest wall tumors are sarcomas accounting for $15-20 \%$ of all sarcomas, categorized according to the 4th edition of World Health Organization (WHO) Classification (2). Due to the low incidence of chest wall sarcoma and lack of high-level clinical evidence, there is no TNM staging about chest wall sarcomas up to now. Currently, only the 8th edition TNM staging criteria of bone tumors (trunk, extremities, skull and maxillofacial) and soft tissue sarcomas (trunk and extremities) can be referenced according to the postoperative pathological diagnosis (3).

Moreover, the staging and treatment principles can refer to the National Comprehensive Cancer Network (NCCN) clinical practice guidelines of bone tumors and soft tissue sarcomas $(4,5)$. The histopathological biodiversity of sarcomas imply different therapeutic approaches depending on the site of origin, the histological grade and histological nature which can be scarcely described by the three factors of TNM. However, most of the studies on which current guidelines have been made up on, mainly focused on bone tumors and soft tissue sarcomas of the extremities, excluding chest wall sarcomas. This is mostly due to the fact that published studies about chest wall tumors include mostly few patients, describe anecdotical surgeries and are almost all single-center retrospective studies. This is the reason why evidences regarding the clinical management of chest wall tumors are lacking, and some controversial issues on surgical and multimodality treatment have yet to be agreed upon.

In January 2019, 73 Chinese experts in thoracic surgery, plastic surgery, science, and engineering jointly released the
Chinese expert consensus on chest wall tumor resection and chest wall reconstruction (2018 edition) (6). Although this consensus has provided clinical guidelines for the diagnosis and treatment of chest wall tumors, numerous experts also put forward new perspectives on some academic issues, pointing out the necessity to further discuss the points of contention. Therefore, we conducted a survey through the administration of a questionnaire to 85 experts to develop a new version of the expert consensus, addressing the controversial points about surgical therapy of chest wall tumors.

\section{Materials and methods}

In 19th May 2021, 62 experts in the world were summoned to Xi'an (Shaanxi Province, China) to discuss the controversial points of the chest wall tumor, and other 23 experts responded by email with their views on the controversial issues. The experts in the study were selected according to the job title, regional distribution, and the independent surgical experience on chest wall tumor. All the controversial questions were distributed to the participants in the form of questionnaires, including rare tumor surgery feasibility, selection of resection margin, reresection scope and standard of timing, chest wall sarcomas staging, reconstruction of chest wall defect, choice of implants, treatment for $\mathrm{T}_{3-4}$ stage non-small cell lung cancer (NSCLC) invading the chest wall, etc. All of results in the questionnaire were summarized and the approve rate of every controversial question was counted. The approve rate was shown in every consensus to represent the opinion of experts on this controversial point. The conclusions were given different levels of recommendation according to the following criteria: category 1 (the expert approval rate is over $80 \%$ ), category 2 (the expert approval rate is $60-80 \%$ ), category 3 (the expert approval rate is $40-60 \%$ ). The new version of the expert consensus about chest wall tumor was formed according the above conclusions. The authors 
are accountable for all aspects of the work in ensuring that questions related to the accuracy or integrity of any part of the work are appropriately investigated and resolved.

\section{Results and discussion}

\section{Consensus 1: Wide excision should be performed for desmoid tumor (DT) of chest wall (category 1). After excluding the distant metastasis by multi-disciplinary team, solitary plasmacytoma (SP) of chest wall can be treated with extensive resection and adjuvant radiotherapy (category 3)}

First described by John Macfarlane in 1832 (7), DTs can be diagnosed in the abdomen, chest wall, scapular girdle, the pelvic girdle, and the distal ends of the lower limbs. Although DTs were identified as benign tumors at the outset due to the rarity of metastasis, they were prone to recur or invade even after wide resection. The pathological features of DTs differ significantly from malignant tumors; however, DTs are regarded as equivalent to low-grade sarcoma due to their tendency towards local invasion and frequent recurrence (8). Therefore, the excision extension of DT has always been an argument of discussion.

DT is a relatively uncommon disease in clinical practice, with $7-10 \%$ of DTs occurring in the chest wall (7). Thus, a standard of care is not achieved yet because many experts hold different positions on the final treatment option and clinical perspective (2-6). In some studies, DTs of the chest wall were excluded from malignant lesions and the patients did not receive wide excision and adjuvant therapy $(8,9)$. However, many investigators consider DT of the chest wall as a low-grade sarcoma, and perform wide excision and chest wall reconstruction for these patients $(7,10-12)$. There are some explanations of these opposing academic views. One of the important reasons is that the margin status and resection thickness was not associated with DT recurrence in numerous studies (13-15). However, since most of the studies were retrospective, single center analyses with a low level of evidence, final conclusions about margin status have not yet been determined in some clinical guidelines (16). In this survey, $80.5 \%$ of surgeons agreed that DT of the chest wall should be treated as a malignant lesion with extensive resection. Wide excision with above $2 \mathrm{~cm}$ margin distance should be attempted to obtain a $\mathrm{R} 0$ resection margin for chest wall tumors. R0 surgical margin should be obtained as far as possible without compromising vital organ function, while R1 surgical margin can only be accepted if there is a risk of serious complications.

$\mathrm{SP}$ is one of the subtypes of multiple myeloma, including osseous and extraosseous diseases. SP is rare, and is characterized by a localized accumulation of neoplastic monoclonal plasma cells, without proof of systemic myelomatosis. SP represents less than $5 \%$ of all plasma cell neoplasms (17) and mostly occurs in the spine, pelvis, and pectoral girdle, while rarely occurring in the sternum, ribs, and clavicle (17-19). According to the current clinical guidelines, radiotherapy with a 40-60 Gy is recommended as an initial standard therapy (20). Surgery is permitted only when local osseous instability or serious nerve compression occurs. However, a few samples with solitary sternum plasmacytoma have previously received wide resection and chest wall reconstruction in clinical practice. Multiple articles present these surgical samples as case reports $(21,22)$. Sabanathan et al. (23) presented 17 SPs in the chest wall treated by radical excision, and seven of these progressed to multiple myeloma within 2 years of surgery. Also, the 5-year survival rate of $59 \%$ was similar to that reported in previous studies $(24,25)$.

Furthermore, a cohort of six patients with solitary sternum plasmacytoma in Tangdu Hospital (Shaanxi Province, China) received wide excision and adjuvant chemotherapy, and obtained a median recurrence-free survival of 36 months (26). Of these patients, three developed to multiple myeloma, and one patient died of the disease. Most of the reported cases that received wide excision are retrospective studies with small sample sizes. Despite complete resection of the local disease, disease free survival was not improved by excision and the long-term survival depended on the subsequent development of multiple myeloma. Due to the lack of highlevel clinical evidence, the role of surgery for chest wall plasmacytoma remains controversial. In this survey, $54.8 \%$ of the surgeons agreed that SP of chest wall should be treated with extensive resection, and $32.1 \%$ of them considered the radiotherapy as the first treatment for SP of chest wall. In addition, $13.1 \%$ of the surgeons were not sure about the issue. A multi-disciplinary team is necessary before surgery, including medical oncology, hematology, radiotherapy. The positron emission tomography-computed tomography (PETCT) and bone marrow puncture must be performed to exclude the distant metastasis of disease before surgery. Given the systemic nature of plasmacytoma, adjuvant radiotherapy is recommended in clinical practice (27). The adjuvant chemotherapy is a controversial issue due to the insufficient clinical evidence and it is recommended only in case of persistent disease on PETCT after radiotherapy (27). 
Consensus 2: Wide excision with above $2 \mathrm{~cm}$ margin distance should be attempted to obtain a $R 0$ resection margin for chest wall tumors (category 1). Given the difficulty of reconstructive surgery for adjacent organs, including the great vessels, heart, trachea, joints, and spine, it is unnecessary to obtain enough resection margin distance if the chest wall tumor involves these vital organs (category 2). However, the tissue adjacent to the chest wall tumor (e.g., a layer of fascia) should be removed as much as possible. As for manubrium sterni tumors, capsula articularis sternoclavicularis can be used as a safe margin marker if the tumor does not invade the joint capsule (category 2)

Radical resection with a $\mathrm{R} 0$ margin is the standard surgical treatment for most tumors. However, the relationship between $\mathrm{R} 0$ resection margin and prognosis of chest wall sarcoma is controversial. In a number of previous retrospective studies (28-31), wide excision of chest wall sarcomas was entirely unrelated to tumor recurrence and patient survival. However, other studies reported that patients with wide excision achieved lower recurrence rates and longer survival (9,32-34). It remains contentious whether wide excision can translate into better survival outcomes for patients, as it often means increased surgical difficulty, postoperative complications, and greater clinical costs. As the above studies were single-center, retrospective, smallsample studies with a low level of clinical evidence, the final conclusions need to be further verified. In this survey, $71.4 \%$ of the surgeons deemed that wide excision was a key predictor of recurrence and survival for patients with chest wall tumors, and $22.6 \%$ of them were not sure whether wide excision can translate into better survival outcomes for patients. Moreover, $6 \%$ of the surgeons considered that wide excision was of no use to the patient. Some potential reasons can be summarized as follows. Multiple pathological types of chest wall tumor result in significant heterogeneity in the previous studies. Also, the pathological types included in previous studies vary greatly, and the skill level of the surgeons varied too. Moreover, the small sample size is also an important factor affecting the research results.

Although most surgeons can agree on $\mathrm{R} 0$ resection for chest wall tumors, the detailed value of surgical margin distance remains controversial. King et al. (33) regarded $4 \mathrm{~cm}$ on all sections of chest wall tumors as a surgical margin distance, and claimed necessity to remove one rib on each of the upper and lower margins of the tumor. The 5 -year recurrence rate of patients in the $4 \mathrm{~cm}$ margin group (29\%) was lower than that of the $2 \mathrm{~cm}$ margin group (56\%), but the difference was not statistically significant. In some studies, patients with a surgical margin of $2 \mathrm{~cm}$ can also achieve a good survival prognosis $(35,36)$. Park et al. believed that patients with a surgical margin of $1.5 \mathrm{~cm}$ could also obtain a good prognosis (32). In clinical practice, the larger the tumor diameter, the more difficult it is to achieve wide excision. Especially for sternal sarcoma, wide excision often requires resection of the adjacent pericardium, thymus, great blood vessels, and other important organs. The larger the defect, the more difficult the reconstruction surgery. Thus, in cases where the reconstructive surgery cannot be performed, including the great blood vessels and heart, a positive surgical margin (R1) is permissible. In these cases, postoperative radiotherapy should be considered.

In this survey, $51.2 \%$ of surgeons believed that the margin distance of $2-3 \mathrm{~cm}$ should be guaranteed during the wide excision. Also, $20.2 \%$ of surgeons thought that a margin distance of $3-4 \mathrm{~cm}$ should be guaranteed, while $13.1 \%$ of them considered that the margin distance of 4-5 $\mathrm{cm}$ should be obtained. Notably, $69.84 \%$ of surgeons deemed that it was not necessary to achieve a sufficient distance on all the tumor margins. R1 margin can be acceptable if the chest wall tumor is located next to vital organs, including the great vessels, heart, trachea, joints, and spine.

As for manubrium sterni tumors, $76.19 \%$ of surgeons believed that capsula articularis sternoclavicularis can be used as a safe margin marker if the tumor does not invade the joint capsule. With respect to the excision of skin adjacent to the tumor, $41.3 \%$ of thoracic surgeons thought that the skin on the surface of the tumor was still widely excised, even though the imaging and palpation examinations did not indicate the invasion of skin, and the excision extension was based on ensuring the incision suture.

\section{Consensus 3: For patients with chest wall tumors undergoing unplanned excision (UE) for the first time, it is necessary to carry out wide excision as soon as possible within 1-3 months following the previous surgery (category 1). Wide excision with above $2 \mathrm{~cm}$ margin distance should be attempted to remove the residual tumor (category 1). Adjuvant radiotherapy or systemic chemotherapy can be performed according to the specific situation of re-resection (category 3)}

It is not uncommon for a surgeon to operate on a presumed benign soft tissue mass without appropriate preoperative imaging or prior biopsy, and then to excise the lesion without attention to the surgical margins. Such surgery has been 
termed an "unplanned excision" or "whoops" procedures $(37,38)$. Most UEs are performed by surgeons without specialty training in oncology, especially in primary hospitals. After unplanned surgery, it is difficult to make the next decision once the patient is diagnosed with a malignant tumor. There is controversy as to whether UEs result in higher recurrence and survival rates. Some previous studies have shown that UEs of local soft tissue sarcomas had no significant effect on disease-specific and metastasis-free survival compared with those with planned excisions $(39,40)$. By contrast, several previous studies emphasized the problems of an UE, showing the higher incidence of local recurrence even after the residual tumor was removed in re-resected specimens (38,41-43). In an analysis of 295 patients with soft tissue sarcoma that underwent expanded re-resection of the tumor bed, residual neoplasms were visible to the naked eye in $28 \%$ of patients, while microscopically-visible residual tumors were present in $46 \%$ of patients $(4,44)$. The 5 - and 10 -year recurrence-free survival rates of patients who underwent re-resection were significantly higher than that of patients who did not undergo expanded re-resection $(4,44)$. However, chest wall sarcoma is a small percentage of these cases; thus, the level of evidence supporting these conclusions is low.

In addition, the optimal time for reresection has not yet been determined. It is difficult to evaluate the anatomical location of the primary chest wall tumor during re-operation, especially the surgical margins of skin and soft tissue, which may lead to misestimation of the resection range. In this survey, $82.2 \%$ of surgeons believed that the re-resection procedure should be carried out within 1-3 months after unplanned resection. Moreover, $73 \%$ of the surgeons believed that a margin distance of $2-4 \mathrm{~cm}$ should be guaranteed during the re-resection procedure, while $16 \%$ of them considered that a margin distance of $4-5 \mathrm{~cm}$ should be obtained. Also, $50 \%$ of surgeons agreed to perform local radiotherapy in the tumor bed or chemotherapy.

Consensus 4: Current tumor-node-metastasis (TNM) staging criteria (American foint Committee on Cancer, AFCC) of bone tumor and soft tissue sarcoma (trunk and extremities) are not suitable for chest wall sarcomas, and it is necessary to develop new staging criteria for chest wall sarcomas (category 2). Depending on the location of the chest wall sarcoma, lymphnode dissection may be performed in the surgically visible area or adjacent mediastinal area (category 3)

Due to the low incidence of chest wall sarcoma and the lack of high-level clinical evidence, there is currently no TNM staging of chest wall sarcoma. Some osseous tumors, including osteosarcoma, chondrosarcoma, Ewing sarcoma, osteoclastoma, must be staged according to the 8th edition TNM staging criteria of bone tumors (trunk, extremities, skull, and maxillofacial) (5). Some soft tissue sarcomas, including leiomyosarcoma, synoviosarcoma, fibrosarcoma, primitive neuroectodermal tumor, hemangiosarcoma and so on, can be staged according to the 8th edition TNM staging criteria of soft tissue sarcomas (trunk and extremities) (4).

However, there are many significant differences between the two staging systems, especially with regards to the $\mathrm{T}$ staging criteria. In the TNM staging criteria of bone tumors (trunk, extremities, skull, and maxillofacial), the tumor is divided into $\mathrm{T} 1(\leq 8 \mathrm{~cm}), \mathrm{T} 2(>8 \mathrm{~cm})$, and $\mathrm{T}_{3}$ (discontinuous tumor), with a cut-off of $8 \mathrm{~cm}$. In the TNM staging criteria of soft tissue sarcomas (trunk and extremities), the tumor is divided into T1 $(\leq 5 \mathrm{~cm}), \mathrm{T} 2(5-10 \mathrm{~cm}), \mathrm{T} 3(10-15 \mathrm{~cm})$, and T4 $(>15 \mathrm{~cm})$, with cut-off of 5,10 , and $15 \mathrm{~cm}$, and also considers tumor location (deep versus superficial) and histological grade. In one of the main references for the establishment of the two staging criteria, sarcomas in extremities $(60 \%)$ were mainly included, while sarcomas in the trunk $(20 \%)$ were encountered less, and there was no grouping of chest wall sarcomas due to the small sample (45).

Notably, the anatomy of the extremities and chest wall differs greatly. Osteogenic and chondrogenic sarcomas in the chest wall with a diameter of $8 \mathrm{~cm}$ often invade $2-3$ adjacent ribs, the pleura, lung, great vessels, and other organs, but the current T1 staging may underestimate the severity of the disease. Referring to the other tumors, it may be inaccurate to determine the extent of tumor invasion only by diameter. In addition, due to the low incidence of lymph node metastases in sarcomas, the current guidelines do not specify lymph node dissection. However, there are numerous lymph nodes surrounding the chest wall sarcomas adjacent to the anterior mediastinum. Although Riad et al. reported lymph node metastasis in $3.7 \%$ of patients with soft tissue sarcomas of the extremities, but lymph node metastasis of chest wall sarcoma has not yet been investigated $(4,46)$.

In this survey, $76.5 \%$ of surgeons believed that the current TNM staging criteria of bone tumor and soft tissue sarcoma were not suitable for chest wall sarcomas and it is necessary to set special staging criteria for chest wall sarcomas. Additionally, $58.7 \%$ of surgeons believed that lymph node dissection should be performed in the visible 
area or adjacent mediastinal area.

Consensus 5: It is necessary to use rigid implants for chest wall reconstruction for chest wall defect exceeds $5 \mathrm{~cm}$ in adults (category 2) and adolescents (category 3). Titanium plate and mesh polymethylmethacrylate are the most commonly used rigid implants. Personalized implants, such as three-dimensional printed implants, have the advantage of anatomically repairing the chest wall defect

Traditionally, chest wall defects larger than $5 \mathrm{~cm}$ in maximum diameter should be reconstructed with rigid implants to prevent chest wall floating, paradoxical breathing, and/or respiratory failure $(47,48)$. The chest wall defects adjacent to the scapula should be reconstructed with rigid implants if the maximum diameter of the defect exceeds $10 \mathrm{~cm}(47,48)$. However, the above views are mainly based on the surgical experience and consensus of clinicians, and there is still a lack of high-level clinical evidence confirming them.

In addition, for adolescent patients with chest wall tumors, the long-term survival should be considered after wide excision, and development of postoperative scoliosis must be afforded greater attention. Several studies have shown that approximately $20-30 \%$ of adolescent patients with chest wall wide excision develop scoliosis in 5-10 years after surgery (49-52). The use of flexible materials such as a mesh for chest wall reconstruction can increase the compliance of the chest wall, which may increase the risk of scoliosis. Therefore, the use of rigid implants for chest wall reconstruction is recommended (52). However, the use of rigid implants for chest wall reconstruction may cause postoperative restriction on the growth of the thorax; thus, many doctors are very cautious about the use of rigid implants for adolescents with chest wall defects.

There are various types of rigid implants in clinical practice, including Matrix-RIB (DePuy Synthes, West Chester, PA), STRATOS (Strasbourg Thoracic Osteosyntheses System, MedXpert GmbH, Heitersheim, Germany), Ribfix Blu (Zimmer Biomet) and Sternalock (Zimmer Biomet), titanium mesh, polymethylmethacrylate, personalized implants, etc. (53-57). The selection of implants is mainly based on the surgical experience, and there are no relevant clinical studies comparing the repair effects of the mentioned implants. The three-dimensional printed (3DP) implants have the advantage of anatomically repairing the chest wall defect, with very good clinical application potential $(56,57)$. Numerous medical centers have carried out chest wall reconstruction using these implants, which is one of the important progresses in the field of chest wall reconstruction in recent years (56-61). Multiple biomaterials can be fabricated as 3DP implants, including titanium, polyether-ether-ketone (PEEK), and polycaprolactone. Titanium is the most common implant material in clinical practice, but the excessive elasticity modulus of titanium may have a stress-shielding effect on bone growth. PEEK has an elastic modulus close to that of cortical bone, but the hydrophobic interface of PEEK may affect bone fusion.

However, 3DP implants come with skill requirements for surgeons and engineers, who require tacit cooperation in the process of implant design, production, and surgery to achieve ideal clinical effects (57). 3DP implants have not yet been approved by the State Food and Drug Administration (FDA) or the National Medical Products Administration of China, and surgery can only be carried out as part of clinical studies in a few medical centers. In this survey, $71.4 \%$ of the surgeons always use rigid implants for chest wall reconstruction if the maximum diameter of chest wall defect exceeds $5 \mathrm{~cm} ; 47.6 \%$ of them use titanium implants, including Matrix-RIB, STRATOS, and titanium mesh, while $15.4 \%$ of them use polymethylmethacrylate and other implants. Also, 50.8\% of thoracic surgeons considered it necessary to use rigid implants to reconstruct chest wall defects in adolescent patients. With respect to the selection of reconstruction materials, $82.5 \%$ of the surgeons had used synthetic mesh and biological mesh, including polypropylene mesh, polylactic acid mesh, nylon mesh, polytetrafluoroethylene (PTFE) mesh, silicone membrane mesh, silicone membrane mesh, bovine pericardium mesh, porcine mesentery mesh, artificial skin, etc.

Consensus 6: For NSCLC invading the chest wall, wide excision is recommended for patients with stage $T_{3-4} N_{0-1} M_{0}$ (category 2). As for NSCLC without lymph node metastasis (stage $T_{3-4} N_{0} M_{0}$ ), the treatment mode of surgery (category 2) + adjuvant chemotherapy (category 3) \pm radiotherapy (category 3) is recommended. As for NSCLC with hilar lymph node metastasis (stage $\left.T_{3-4} N_{1} M_{0}\right)$, the treatment mode of neoadjuvant chemotherapy (category 2) \pm radiotherapy (category 2) + surgery (category 2) is recommended

NSCLC invading the chest wall is a rare clinical disease. According to the TNM staging criteria in the 8th edition 
of the NCCN NSCLC Clinical Practice Guidelines, NSCLC invading the chest wall can be classified as stage $\mathrm{T}_{3}$ or $\mathrm{T}_{4}$. A number of retrospective clinical studies have shown that patients with NSCLC invading the chest wall can benefit from surgery, especially those without lymph node metastasis (62-69). Patients with NSCLC invading the chest wall (stage $\mathrm{T}_{3-4} \mathrm{~N}_{0} \mathrm{M}_{0}$ ) can achieve a 5 -year survival rate of $30-67 \%(62-69)$. However, most of the above studies were retrospective, single-center, and smallsample studies with a low level of clinical evidence. Thus, in the NCCN and Chinese Society of Clinical Oncology guidelines, surgery is only recommended as level 2 evidence for patients with NSCLC invading the chest wall (stage $\mathrm{T}_{3-4} \mathrm{~N}_{0} \mathrm{M}_{0}$ ) (70).

$\mathrm{R} 0$ resection and lymph node invasion are the major prognostic factors for NSCLC invading the chest wall. In this survey, $76.2 \%$ of surgeons agreed to carry out a wide excision for patients with NSCLC invading the chest wall without lymph node metastasis (stage $\mathrm{T}_{3-4} \mathrm{~N}_{0} \mathrm{M}_{0}$ ), and $50.8 \%$ of them continued to administer adjuvant chemotherapy and/or radiotherapy. Also, $66.6 \%$ of surgeons agreed to perform a wide excision for patients with NSCLC invading the chest wall with hilar lymph node metastasis (stage $\mathrm{T}_{3-4} \mathrm{~N}_{1} \mathrm{M}_{0}$ ), while $62.6 \%$ administered neoadjuvant chemotherapy and/ or radiotherapy preoperatively. Only $17.5 \%$ of surgeons considered operating on patients with NSCLC invading the chest wall with mediastinal lymph node metastasis (stage $\mathrm{T}_{3-4} \mathrm{~N}_{2} \mathrm{M}_{0}$ ). Wide excision with above $2 \mathrm{~cm}$ margin distance aiming for R0 resection should be attempted to achieve an en bloc resection.

\section{Key questions and perspectives}

Question 1: Although the DT was identified as benign tumor at the outset due to the rare metastasis, it was prone to recur or invade even after wide resection. The pathological feature of $D T$ is significantly different from the malignant tumor, however, DT was regarded as equivalent to low-grade sarcoma because of the tendency to local invasion and frequent recurrence. Should extensive surgical resection be performed for DT?

Karel W. E. Hulsewé: Yes.

Yvonne L. J. Vissers: Yes.

Erik R. de Loos: Yes.

Giuseppe Marulli: Wide local excision is the most effective treatment, by including at least one unaffected rib above and below the lesion, as well as intercostal muscles, pleura, and a wide clear margin of adjacent soft and osteal tissues $(7,12,71)$. Wide (R0) microscopic margins resection should be the goal, with a localized control rate of $85 \%$.

In some cases, positive (R1) microscopic margins can be accepted when radical excision may be difficult [the process involves paravertebral structures, the spine, the brachial plexus, great vessels, or extends into the soft tissues of the neck (72-74)] or when palliative surgical diminution is performed as a life-saving treatment when compression of vital organs is present (71).

However, if positive microscopic margins can be anticipated, other managements than surgery should be preferred. In addition, if R1 resection is obtained in first-line management, there is insufficient evidence to recommend either perioperative radiotherapy or reoperation. Although the risk of a local recurrence seems to be lower after combined modality, the difference between surgery alone and surgery plus perioperative radiotherapy is not statistically significant (75).

Alberto Sandri: DT of the chest are rare and should be considered border line tumours due to a high recurrence rate and its possible genetical asset. Aggressive surgery should be the mainstay of treatment aiming at an R0 resection. Careful preoperative assessment and planning may help in defining the extent of surgery (CT scan, RMN). The free margin should be of at least $2-3 \mathrm{~cm}$ with a full thickness resection if possible, in consideration of tumours' proximity with (vital) organs. If R0 resection cannot be achieved, then a multimodal approach is mandatory either in the neoadjuvant (down staging) or adjuvant settings (RT+/CT). Routine surveillance is mandatory for many years after surgical treatment for possible disease relapse.

Andrea Bille: Considering the increased risk of local recurrence yes.

Jin Yong Jeong: Abbas et al. (12) performed radical resection of chest wall DTs in 53 patients, complete resection in 44 cases and incomplete resection in 9 cases. The recurrence rate was $89 \%$ in incomplete resection and $18 \%$ in complete resection. As in this report, if resection is incomplete, the recurrence rate is very high, so I believe that extensive resection should be performed.

Luca Ampollini: I think that wide surgical resections should be performed for DT even if the margin status of surgery was not associated with DT recurrence in numerous studies. The event of tumor recurrence could be quite challenging. For this reason, mostly for young patients, 
wide excisions and chest wall reconstruction should be carried out. Frequent recurrences have been reported even in case of previous macroscopic resection.

Masatsugu Hamaji: I agree in that wide radical resection should be attempted as a first-time procedure whenever possible, whereas at recurrence lesser resection should be considered given potential re-recurrences.

Yuichiro Ueda: Surgical treatment of primary DT of the chest wall requires wide local resection because local recurrence develops frequently. In cases of recurrence or inadequate resection, multimodality therapies should be considered. In my experience, distant metastasis of DT is rare.

Zsolt Sziklavari: I do not agree with your opinion. Desmoid fibromatosis is a locally aggressive but nonmetastasizing deep-seated (myofibroblastic) neoplasm with infiltrative growth and propensity for local recurrence. According to WHO, although primary surgery with R0 was classically considered to be the standard of care, local recurrence does not consistently correlate with margin status. Thus, function-sparing surgery should be preferred to aggressive surgery seeking negative margins. In patients with recurrence or gross residual disease, radiotherapy is effective for local control. A re-resection is also possible, but it can be morbid. With or without recurrence, no patient should die of the disease. Tumor-related deaths are rare but seem to be more common in patients with familial adenomatous polyposis. In my mind, the extensive surgical resection (R0-max.) is not necessary. I advise R0.

Jacopo Vannucci: This tumor represents a particular case. In my opinion, the margin must be considered the first parameter for cure. Considering the current knowledge on this tumor and its treatment (current best of practice), the margin should be clear and wide when possible. If the tumor is located in a difficult position, the best option is to balance morbidity and radicality.

Alfonso Fiorelli: R0 resection is the main goal of surgical treatment of desmoid chest wall tumor. However, when the tumor invades vital structures or in cases of large tumors, obtaining $\mathrm{R} 0$ resection is technically challenging. In these particular cases, the objective should be to preserve the organ function and the patients' quality life rather than striving for R0 resection. In contrast to most malignant tumors, the prognostic significance of microscopically positive margins remains controversial for desmoid chest wall tumors. In several series the lack of R0 was not associated with an increased risk of local recurrence. Brodsky et al. (76) reviewed the clinical data of 32 patients undergoing resection of chest wall DT at Memorial SloanKettering Cancer Center. Gender, tumor site, tumor greater than $10 \mathrm{~cm}$ and incomplete resection were not associated with significantly increased local recurrence rates while age greater than 30 years at presentation was correlated with a significantly reduced prevalence of local recurrence. Abrão et al. (77) retrospectively reviewed 19 patients undergoing surgical resection of chest wall DT. Positive margins and the resection thickness were not significant risk factors for a later recurrence. Lev et al. (78) retrospectively evaluated the outcomes of 146 patients who underwent resection of DTs at the M.D. Anderson Cancer Center. The authors found that patients with microscopically positive or negative margins had no differences in their long-term recurrence rates.

In patients with macroscopic residual disease after surgery, adjuvant radiation therapy in combination with surgery may result in equivalent local control to surgery with negative margins.

Beatrice Aramini: DT is a rare and locally aggressive monoclonal, fibroblastic proliferation characterised by a variable and often unpredictable clinical course. Previously surgery was the standard primary treatment modality; however, in recent years a paradigm shift towards a more conservative management has been introduced and an effort to harmonise the strategy amongst clinicians has been made (75).

"Surgery may still be considered as a second-line treatment for AW tumours as morbidity and risk of recurrence are limited, while other modalities should be preferred for DT located at other sites. Clearly, patients need to be referred to centres with experience in DT to minimize the risk of active surveillance and avoid unnecessarily debilitating or mutilating surgery when possibly needed. Surgery by surgeons without significant experience in the management of DT is strongly discouraged. Similarly, referral to experienced multidisciplinary teams is recommended at the time of initial diagnosis, for optimal advice on safety of an initial observation strategy".

Cecilia Pompili: /.

Chaozong Liu: As DT is a benign tumor and has a tendency to local invasion, I don't think extensive surgical resection can stop its recurrence. The extensive surgical resection may potentially have impact on patients recovery.

Fabio Davoli: DT has to be considered as a soft tissue tumor so it always requires extensive surgery with radical purposes. A further consideration is mandatory about the primary site of the DT. If the DT belongs to the abdominal 
wall, surgery is the first line of treatment, then followed by adjuvant chemotherapy. If DT belongs to the chest it is controversy if neoadjuvant chemotherapies have to be administered and surgery reserved only if a good response is documented.

Hans Van Veer: Yes, first resection should have curative intent including R0.

Hiroaki Kuroda: /.

Ilhan Inci: In my opinion, extensive surgical resection should be performed for DT.

Inderpal S. Sarkaria: /.

Ricciardi Sara: An accurate pre-operative plan should be done (useful 3D reconstruction models). R0 surgical resection is sufficient in case of DT, in case the $\mathrm{R} 0$ resection seems to compromise vital organ, consider R1 resection + adjuvant treatment if indicated.

Servet Bölükbas: Yes, in order to avoid recurrence.

Question 2: SP is one of the subtype of multiple myeloma, including the osseous and extraosseous disease. According to the current clinical guidelines (20), radiotherapy with a 40-60 Gy is firstly recommended as a standard therapy. Surgery is permitted only when local osseous instability or serious nerve compression occurs. However, a few samples with solitary sternum plasmacytoma received wide resection and chest wall reconstruction in clinical practice. Should extensive surgical resection be performed for $S P$ ?

Karel W. E. Hulsewé: No as a standard treatment; only on indications as mentioned and then as sparingly as possible with consideration adjuvant radiotherapy.

Yvonne L. J. Vissers: No, only when local osseous instability or serious nerve compression occurs.

Erik R. de Loos: No.

Giuseppe Marulli: Radiotherapy is the treatment of choice for SP, while extensive resection is controversial because of the rarity of the disease and of the rarity of surgical indications. Wide excision is a treatment option for tumors that are completely resectable, when there are tumor-induced symptoms (severe pain, skeletal instability, or pathologic fracture) and for distinct localizations (spine with neurological damage, upper airway that cannot be treated with radiotherapy, or vertebral fractures that require stabilization) $(79,80)$.

Few case reports, extremely anecdotical, can be found in literature describing surgical resection of SPs originating in ribs and in the sternum. Moreover, despite complete resection of the local disease, the long-term survival depended on the subsequent development of multiple myeloma.

Alberto Sandri: As per NCCN multiple myeloma guidelines, 30-60 Gy RT should be the first treatment with surgery as an occasional treatment in case of bone instability, nerve involvement.

Andrea Bille: The literatures recommend RT as first treatment for solitary SP, but in case on symptoms or chest wall deformity surgery should be considered as a valid option (20).

Jin Yong Jeong: Radiation therapy is the primary treatment for SP, and surgical treatment is controversial. Sabanathan et al. (23) reported that SP of the chest wall was radically resected in 17 patients, 23 of whom had progressed to multiple myeloma, and suggested that surgical resection might prevent systemic dissemination. Advances in chest wall reconstruction techniques allow extensive resection of SP of the chest wall as an alternative primary treatment or an effective debulking procedure to reduce the morbidity of high-dose radiation therapy (24). Based on these evidences, if SP resection is performed, I agree to extensive resection of the chest wall.

Luca Ampollini: Multidisciplinary approaches including radiotherapy, surgical resection, and chemotherapy, should be always discussed when facing with these rare tumors. Most of the time, radiation therapy is determined to be the best choice for the patient. In the era of personalized medicine, I would consider a subsequent resection in case of single bone disease.

Masatsugu Hamaji: Since plasmacytoma origins in bone marrow, even extensive resection cannot guarantee microscopic complete resection. If a lesion is limited, extensive resection should be accompanied by adjuvant radiotherapy.

Yuichiro Ueda: Unfortunately, I have no experience of SP.

Zsolt Sziklavari: Solitary bone plasmacytoma (SBP) is defined as the clonal proliferation of plasma cells identical to those of plasma cell myeloma, which manifests itself as a localized osseous growth. The most common chest wall location is the ribs, followed by the clavicle and sternum. The role of surgery is to establish the diagnosis by biopsy. Most lesions appear circumscribed and osteolytic without extremely infiltration into adjacent tissue, so that R0 is sufficient. Local recurrence is uncommon for plasmacytomas. After they are treated with radiation therapy, in $35-55 \%$ of patients multiple myeloma develops. That is why no R0-max has to be done. In case of instability 
or pain, a secondary R0 resection can be performed. An extensive surgical resection (R0-max.) is not necessary in my point of view.

Jacopo Vannucci: This issue is unsolved and the question cannot be answered with a yes/no. The treatment must have a systemic perspective considering the disease and its possible evolution. As surgery proved to have a potential success in selected cases, a tumor resection with radical intent should be taken into account in particular conditions. In such cases, a multidisciplinary teamwork is the key factor for a successful therapeutic schedule. Surgery needs to aim at radical removal but represents a single passage of a multimodal treatment.

Alfonso Fiorelli: Surgery may have a role for management of SP as a part of multimodality treatment. Recent advances in the techniques of skeletal and musculocutaneous reconstructions have facilitated the treatment of these tumors so that tumor size is not a contraindication to radical excision. Radical en-block resection of the tumor can decrease the morbidity of large doses of radiotherapy either preoperatively or postoperatively.

Beatrice Aramini: SP is an infrequent form of plasma cell dyscrasia that presents as a single mass of monoclonal plasma cells, located either extramedullary or intraosseous. In some patients, a bone marrow aspiration can detect a low monoclonal plasma cell infiltration which indicates a high risk of early progression to an overt myeloma disease. For decades, treatment has been based on highdose radiation, but studies exploring the potential benefit of systemic therapies for high-risk patients are urgently needed.

In many instances, patients have had surgery, with complete or partial tumor removal, as part of the diagnostic procedure. Apart from the diagnostic approach, the indication of surgery is fixation of fractures, decompressive laminectomy, or spine stabilization. The introduction of modern spinal fixation and stabilization methods, such as vertebroplasty and kyphoplasty, allows for a surgical solution for patients who develop vertebral fractures, vertebral instability, neurological complications, or a combination of these. Radiotherapy can be delayed until after surgery but it is still required because tumor excision without subsequent radiotherapy results in a very high rate of local recurrence (27).

Cecilia Pompili: /.

Chaozong Liu: extensive surgical resection could be considered, but special care must be taken to avoid damage to the nerve.

Fabio Davoli: My answer to question 2 is yes, SP should undergo extensive surgical resection and chest wall reconstruction.

To be honest, in my experience I deal with a SP of the lung only, never with a SP of the chest wall. Regarding sternum plasmacytoma suitable to surgical resection it is a rarity, but it is described. Primary sternal plasmacytoma could be one of the surgical indications to sternal allograph transplantation, as well as chondrosarcomas; only a few cases are described in literature and this does not allow us to reach definitive considerations.

Hans Van Veer: Only when breakthrough through sternal cortices, where radiotherapy would as such not achieve local control eg. When the layer towards the skin becomes too small.

Hiroaki Kuroda: /.

Ilhan Inci: I suggest extensive surgical resection: wide resection and reconstruction if the patients' general condition tolerates this option.

Inderpal S. Sarkaria: /.

Ricciardi Sara: In case of resectable tumors, in young and fit patients, surgical option can be considered but only after MDT evaluation and in the context of multimodality treatment in high volume centers. The sternal reconstruction can be a challenging procedure, a careful preoperative plan should be made.

Servet Bölükbas: Yes. Solitary plasmocytoma have the ability to destruct the sternum. There is a risk of instability and severe bleeding.

Question 3: $R 0$ resection margin is the standard of surgical treatment for most tumors. However, the relationship between $R 0$ resection margin and prognosis of chest wall sarcoma has been controversial. In a number of previous retrospective studies, wide excision of chest wall sarcomas was entirely unrelated to tumor recurrence and patient survival (28-31). But in other studies, patients with wide excision have been shown to obtain a lower recurrence rates and longer survival (32-34). Whether wide excision can translate into better survival outcomes for patients? As it often means more surgical difficulty, more surgical complications, and greater clinical costs

Karel W. E. Hulsewé: The aim is to obtain a R0 resection with a margin of approximately $1 \mathrm{~cm}$ unless this involves critical structures in which case less margin can be accepted.

Yvonne L. J. Vissers: No.

Erik R. de Loos: The aim of surgical treatment should be to strive for a $\mathrm{R} 0$ resection. 
Giuseppe Marulli: /.

Alberto Sandri: It is difficult to express an unbiased opinion on such a topic due to the fact that basically all of the literature dealing with such matter rely on single institution retrospective series, with data from the late 80's-90's.

However, all data point out that R0 surgery for primary tumours of the chest wall is the goal standard affecting the OS, and contemplates (if anatomically possible) wider resections. If this is not achievable, a multimodal approach is mandatory.

Wider excisions are considered for an R0 (margin free) resection but, if achievable, it can affect the reconstruction and may translate in complications and poor quality of life. Information in literature on this topic is scarce. This should be discussed with the patient after a multidisciplinary decision has to be taken. If an MDT-patient consensus is achieved, this can be undertaken.

Andrea Bille: I think the aim should be for Ro resection.

Jin Yong Jeong: Although the relationship between R0 resection and the prognosis of chest wall sarcoma is controversial, I believe that R0 resection is necessary for surgery. Although extensive resection has a high clinical cost, I believe that the difficulties and complications of surgery can be overcome with advances in surgical techniques and biomedical technologies.

Luca Ampollini: Primary chest wall sarcomas are rare tumors. From a therapeutic point of view, they are considered challenging tumors. This complexity underscores the importance of a multidisciplinary team approach to the diagnosis, treatment, and supportive care of patients chest wall sarcomas. A team approach is important at all points: from diagnosis, through treatment, to end-of-life care. It also needs to be patient-centered and must involve the patient in decision-making concerning treatment. These patients should be referred to high volume centers in order to optimize the treatment related costs and to minimize postoperative complications.

Masatsugu Hamaji: Due to the rarity of the disease, rigorous statistical analysis appears challenging to identify prognostic factors. Currently I am doubtful as to whether extensive resection will translate into more favorable longterm outcomes.

Yuichiro Ueda: I partially agree with wide excision because some chest wall tumors can manage surgical resection. In contrast, high-grade cancer and larger chest wall tumor often develop distant metastasis. Therefore, adjuvant therapy is important.
Zsolt Sziklavari: The results after thoracic wall resection are interpreted controversially, because of the very heterogeneous histological findings. Soft tissue sarcomas (fibrosarcomas, liposarcomas, malignant fibrous histiocytomas, rhabdomyosarcomas, dermatofibrosarcoma protuberans, angiosarcomas) represents nearly 50\% all the primary chest wall tumors. The difficulty in treating this type of neoplasm is related to the significant incidence of local recurrence with or without primary R0 status. R1 negatively affect both the disease-free survival and overall survival rates, therefor the wide $\mathrm{R} 0$ resection of primary malignant chest wall sarcoma seems to be absolutely necessary. Opinions differ as to what constitutes wide resection. In our institution all chest wall tumors undergo primary resection with the involved adjacent structures. The resection includes at least $2 \mathrm{~cm}$ "safety" margin of macroscopic normal tissue on all sides (it means macroscopic R0 border plus $2 \mathrm{~cm}$ verified by cryosection). Of course, high-grade malignancies also need to have the entire involved bone resected, because those tumors have the potential to spread within the marrow cavity or along tissue planes (in such cases frozen section can be extremely helpful). A follow-up and a re-resection are possible.

Jacopo Vannucci: The current scientific knowledge and clinical practice suggest to perform a wide excision of the sarcoma. This indication is supported by several analyses that suggest this approach. Considering the local condition, the resection margin is rarely the same in all perimeter. When the resection can be performed achieving a radical resection, the margin should be adapted to the proximal organs, eventually sacrificing the distance for safety. Possible complications, organ failure or instability after reconstruction are sufficient conditions to reduce the resection margin. In case of intraoperatively verified impossible radicality, to abort the resection instead of leaving a positive margin after huge chest wall defects should be considered.

Alfonso Fiorelli: The mainstay of treatment of chestwall sarcoma is wide local excision with radiation with or without chemotherapy. Alternatively, low-grade, small $(<5 \mathrm{~cm})$, subcutaneous tumors may be treated with wide local excision alone. With these methods of treatment, local control rates are excellent $(28,35)$. Induction chemotherapy is not recommended in cases of resectable tumors while adjuvant chemoradiation is associated with a reduction in disease-free survival when added to surgery alone (81).

Beatrice Aramini: There have been several large studies 
that analyzed the prognostic significance of surgical margins. Unfortunately, they presented inconsistent results questioning the prognostic impact of the quality of surgery. The biology of the tumor might ultimately dictate the outcome; however, given the diminished outcome of patients left with positive margins, surgical efforts should aim to achieve microscopically clear margins whenever feasible. Here, only the quality of surgical margins and not the negative margin width attained appears to have an influence on survival. A radical surgical approach with the goal of wide negative margins cannot be justified by the current data.

Cecilia Pompili: /.

Chaozong Liu: With the advancement of imagingguided surgical technology and surgical instrumentation, wide excision could be considered. It not necessarily means increase the surgical difficulty and complications.

Fabio Davoli: The prognostic impact of surgery in chest wall sarcomas is mainly conditioned by two factors: the histopathologic type of sarcoma and the role of neoadjuvant chemotherapy. The resection must be always wide to ensure more chances to the patient.

Osteosarcomas of the chest wall are relatively rare tumors; survival is related to the extent of the resection, but mainly to the chemosensitivity of the tumor. Ewing sarcoma is also mainly related to chemosensitivity and surgical resection both.

Focusing on the specific question my answer is yes.

Hans Van Veer: Being cancer surgery, R0 resection needs to be thrived for, as these tumors do not respond very well to other treatment modalities in case of recurrence. Indeed, this could encompass a large resection involving also an extensive reconstruction and dito costs. However, in case of recurrence because of a too small resection margin, most likely the needed resection space will be even larger, on top of the already resected area.

Hiroaki Kuroda: /.

Ilhan Inci: R0 is always important but it should not jeopardize patient.

Inderpal S. Sarkaria: /.

Ricciardi Sara: Chest wall sarcoma should be treated with wide $\mathrm{RO}$ resections as, as far as I know, those histology poorly respond to chemo or radiotherapy. If surgery is feasible, an R0 resection should be definitely obtained.

Servet Bölükbas: Until we have more convincing data, we have to follow the rules of oncologic surgery. The most important prognosticator for solid tumors is complete resection (R0).
Question 4: Although most surgeons can agree on R0 excision on chest wall tumor, the detailed value of surgical margin distance is still controversial. Michael King et al. regarded $4 \mathrm{~cm}$ on all sections of chest wall tumor as a margin distance in surgery, and it is necessary to remove one rib on each of the upper and lower margins of the tumor (33). The 5-year recurrence rate of patients in $4 \mathrm{~cm}$ margin group (29\%) was lower than that in $2 \mathrm{~cm}$ margin group (56\%), but the difference was not statistically significant. In some studies, the patients with a surgical margin of $2 \mathrm{~cm}$ can also achieve a good survival prognosis $(35,36)$. Ilkun Park et al. believed that the patients with a surgical margin of $1.5 \mathrm{~cm}$ could also obtain a good prognosis (32). What is the reasonable surgical margin distance for chest wall tumor?

Karel W. E. Hulsewé: It does not seem to be expected that sarcomas of the chest wall have a different behaviour compared to other localizations. Therefore a R0 resection with a margin of approximately $1 \mathrm{~cm}$ should be aimed for.

Yvonne L. J. Vissers: En bloc excision of the tumor aiming for a rim of 1-2 $\mathrm{cm}$ of normal uninvolved tissue surrounding it suffices; $\mathrm{R} 0$ tumor free margins are adequate.

Erik R. de Loos: 2 centimeters.

Giuseppe Marulli: Achieve R0 margins as this is associated with a lower local recurrence rate compared to $\mathrm{R} 1$ or $\mathrm{R} 2$ margins $(28,35,82)$.

A recent meta-analysis by Sacchetti et al. (83) proved that the odds ratio (OR) to develop a local recurrence in a 5-year period was significantly in disfavor of patients in which a tumor residual was found in the excision bed $(\mathrm{OR}=3.36$; $95 \%$ CI: 1.97-6.44; $\mathrm{P}<0.001)$. Patients with residual tumor also faced higher risk to develop a distant metastasis $(\mathrm{OR}=3.42$; 95\% CI: $2.54-5.01 ; \mathrm{P}<0.001)$ and lower overall survival $(\mathrm{OR}=2.26$; 95\% CI: 1.63-3.14; $\mathrm{P}<0.001)$.

King et al. (33), who published the first report on the importance of a wide surgical resection margin for primary chest wall sarcoma, defined wide resection as a margin of $4 \mathrm{~cm}$ with several partial ribs above and below for rib tumors and entire bone resection for sternal or manubrial cancers.

In high-grade chest wall sarcomas, resection should follow the following criteria:

(I) Margin greater than $4 \mathrm{~cm}$;

(II) Remove one rib on each of the upper and lower margins of the tumor.

In case of preoperative chemotherapy being administered, the margin should be chosen in function of the pathological response:

(I) The lesion responds to preoperative chemotherapy 
a minimum of an adequate wide margin of $2 \mathrm{~cm}$ should be used;

(II) The lesion does not response to preoperative chemotherapy a margin greater than 3 or $4 \mathrm{~cm}$ wide should be selected.

In low-grade chest wall sarcomas and in chest wall sarcoma located near vital structures, a minimum margin of $2 \mathrm{~cm}$ should be used.

Alberto Sandri: A $4 \mathrm{~cm}$ resection margin with resection of the upper and lower ribs should be considered the standard for resection for primary chest wall tumours until further prospective studies will show different results.

Andrea Bille: I think $4 \mathrm{~cm}$ should be the ideal margin.

Jin Yong Jeong: When resecting a chest wall tumor, it is considered that the resection is performed with a safety margin of $2 \mathrm{~cm}$ or more. However, I believe that there is still controversy about the reasonable surgical margin distance for chest wall tumor as the resection margin distances vary between the reported papers.

Luca Ampollini: Surgical resections should be carefully planned including the aspect of surgical margins. The choice of tumor margin distance should be based on tumor biology, tumor site, tumor dimension, and invasion or proximity with vital organ and structures. As the word itself suggests, a reasonable choice is based on coherent, logical and rational evaluations considering all patient and tumor characteristics and, ultimately, should be chosen by the surgical team during the operative procedure. The final choice should be in accordance with all the above mentioned aspects related to the tumor, should be not extreme or excessive, should not be wasteful. Since it's a challenging decision making process, multiple team members are required. Multidisciplinary care offers not only significant benefits to patients, but also many advantages to the team, including promotion of evidencebased care and opportunities for both continuing educational and quality assurance.

Masatsugu Hamaji: This topic appears to have an overlap with the previous topic. In most cases, I consider $2 \mathrm{~cm}$ margin is sufficient.

Yuichiro Ueda: This is a difficult question, but I think the margin distance of approximately $2 \mathrm{~cm}$ should be obtained. The surgical margin should be determined by not only CT but also MRI or PET-CT. Moreover, surgeons should confirm the margin using a thoracoscope.

Zsolt Sziklavari: See A3. After fixed tissue processing was evaluated re-resection can be done if necessary.

Jacopo Vannucci: This issue of margin distance is matter of debate since a $4-\mathrm{cm}$ distance is usually very difficult to be achieved. Radical surgery should aim at performing a resection margin as wider as possible (up to $4 \mathrm{~cm}$ margin in high grade tumor) without exceeding into unsafe procedure.

Alfonso Fiorelli: An adequate resection margin distance is a well-known prognostic factor for recurrence after chest wall sarcoma resection. The length of margin resection depends by different factors including the tumor size, tumor site, and the characteristics of the tumor. King et al. (33) reported that a 2-cm margin would be safe for low-grade sarcomas, such as chondrosarcoma, while 4-cm margin is indicated for highgrade sarcoma. Wouters et al. (35) described a 1- to $2-\mathrm{cm}$ margin of normal tissue as their principle of surgical resection for primary chest wall sarcoma.

To avoid inappropriate measurements during operation, surgeon should measure the resection margin distance in all directions rather than considering only 1 of the 4 directions.

Beatrice Aramini: Anterior chest wall resections may significantly alter the respiratory physiology depending on whether the entire sternum is involved, the size of the defect, and the type of reconstruction. Meticulous preoperative planning is necessary to effect radical resection and reconstruction according to the principles of biomimesis. To this end, computed tomography image reconstruction and digital subtraction are increasingly becoming a mandatory adjunct to visualize the extent of the chest wall involvement with special attention to tumor margins. To complete preoperative evaluation of tumors infiltrating the full thickness of the chest wall, a video-assisted thoracoscopic assessment of the inner chest wall can be useful, especially when chest wall recurrences from breast cancer or residual tumor after chemotherapy are to be assessed. Because frozen section is not feasible on bone specimens, up to $4-$ to $5-\mathrm{cm}$ tumor-free margins should be obtained.

Although the decision about reconstructive strategy should be individualized, it is common knowledge that the defect resulting from the removal of only one rib may not necessitate reconstruction. However, anterior one-rib defects (i.e., rib 4 or 5) in selected patients may require covering (i.e., young athletes) if there is a consistent risk for lung herniation.

For malignant primary chest wall tumors, the immediate adjacent ribs should be resected for the corresponding length of the infiltrated segment on tumor-free margins.

When the manubrium and one or both clavicles are involved, these can be removed without subsequent reconstruction, yielding acceptable shoulder girdle movement.

Time-honored materials for chest wall reconstruction (i.e., polypropylene, polyglactin meshes, or methylmethracrylate 
sandwich (MMM) along with polytetrafluoroethylene (PTFE) patches) are still valuable options albeit new materials (i.e., titanium plates, acellular collagen matrix meshes, and cryopreserved homografts) are increasingly being preferred due to resilience to infection and ready incorporation into the host tissues.

Cecilia Pompili: /.

Chaozong Liu: The margin depends on the tumor size, grade, patients age and locations. Higher margin could be considered if patients health allowed.

Fabio Davoli: In my experience a margin distance more than $2 \mathrm{~cm}$ is enough to ensure a good prognosis; to remove one rib on each of the upper and lower margins is mandatory also.

Hans Van Veer: I would state a margin of at least $2 \mathrm{~cm}$; most likely this will as such involve also resection of the adjacent rib, keeping in mind the $3 \mathrm{D}$ built of the chest wall.

Hiroaki Kuroda: /.

Ilhan Inci: For margin it is also important where the tumor is localized. For my practice it should be at least $2 \mathrm{~cm}$.

Inderpal S. Sarkaria: /.

Ricciardi Sara: In my clinical practice, I apply $2 \mathrm{~cm}$ of safe margin in chest wall tumors resection.

Servet Bölükbas: Until we have more convincing data, I would plea for $4 \mathrm{~cm}$. Not rarely, discontinuing tumor spread can be found at the resection margins. During surgery, frozen section is not possible to examine the osseous resection margins. More safety margin should translate in more complete resections.

\section{Question 5: For patients with chest wall tumor undergoing UE for the first time, is it necessary to carry out the wide excision as soon as possible? How long can the wide excision be performed after the first surgery?}

Karel W. E. Hulsewé: On the one hand swift reintervention is to be commended but reintervention in the first few weeks may have increased risk of postoperative infections. In the absence of valid data I would suggest to postpone the reintervention for 4 weeks assuming wound healing is not complicated.

Yvonne L. J. Vissers: 1-3 months.

Erik R. de Loos: If suspected malignancy: yes. The optimal time interval between initial and secondary surgery is unclear. In general, edema and other soft tissue reactions will be disappeared after approximately 4 weeks.

Giuseppe Marulli: UEs are defined as tumor excisions without an appropriate diagnosis, preoperative imaging or planning with an estimate incidence up to $18-66 \%$ (83). Patients with positive and uncertain margins after primary surgery should have secondary resection as soon as possible (84). Systematic re-excisions are still representing the gold standard of treatment in patients who underwent unplanned resections of sarcoma. In this setting, these patients undergo a tumor bed excision generally within 3 months from the inadequate surgery even if there aren't any radiological or clinical evidences of local recurrences.

Recently, a wait and see approach was reported to be a viable alternative in selected patients and in referral centers to systematic re-excision after unplanned surgeries (85), postponing re-excisions until a clinically evident local recurrence is detected based upon the assumption that local control do not have influence on overall survival in patients affected by soft tissue sarcomas (86).

Alberto Sandri: Unplanned resections should occur in the least number of cases as possible in order to overcome such problem.

However, in case this occurs, an UE for a suspected primary tumour of the chest wall, the surgeon should treat the lesion as such and therefore keep $2-4 \mathrm{~cm}$ wide margins, possibly avoiding resections of the superior and inferior ribs. In case the R0 resection is not achieved, re-surgery should be adequately planned in the attempt of radicality.

Andrea Bille: Within 6 to 8 weeks max.

Jin Yong Jeong: /.

Luca Ampollini: As mentioned before, I believe that unplanned resection should be avoided. In front of a chest wall tumor, a diagnostic biopsy should be always performed in order to plan the optimal surgical strategy. In the absence of a histological diagnosis, I would not decide to perform a wide excision and reconstruction since it might be an overtreatment that may lead to problems and harmful side effects. With regard to the second point, the diagnostic histological examination should be available within two weeks, depending on immunohistochemical analysis and additional requests (mutations, ...). After obtaining that crucial information, a wide excision should be performed in a reasonable timeframe, that might be estimated at two weeks. And, even then, the final decision should be shared with other team experts. High volume centers, also in view of the rarity of these tumors, possess the appropriate and adequate resources to offer an optimal therapeutic strategy to patients.

Masatsugu Hamaji: I personally do not have a similar situation to the above, but I would say we should carefully discuss pathological findings in the tumor board conference, 
rather than rash to a second resection for obtaining a wider margin.

Yuichiro Ueda: Depending on the patient's condition, wide excision is recommended within 1 month.

Zsolt Sziklavari: Generally, any attached structures, such as the lung, thymus, fat, pericardium, or chest wall muscles have to be excised. If you wait with the excision after biopsy, you cannot distinguish between scar and tumor. We recommend to perform the resection as soon as possible. See also A3-4.

Jacopo Vannucci: In these cases, the margin distance might be considered secondary if the local condition is unknown, poorly assessed or undiagnosed. However, if a wide resection is possible without high risk for functional chest wall defect and mediastinal organs dysfunction, it should be considered and performed. According to a few recent contributions, a watchful patient control is suggested for unsatisfactory margin in some selected cases. Some other Authors suggest a second operation which is performed in a couple of months. Adjuvant treatment must be discussed very soon.

Alfonso Fiorelli: Because the majority of soft tissue sarcomas originating from the chest wall are superficial and their resection is easily achieved, UE is frequently performed. Furthermore, due to the anatomic characteristics of the chest wall, it is often difficult to achieve an adequate resection margin in cases of wide resection. To avoid it, the guidelines from the National Comprehensive Cancer Network (NCCN) indicate that management of soft tissue sarcoma requires appropriate workup which includes localized and systemic imaging, carefully planned core needle or incisional biopsy, and discussion in a multidisciplinary setting (16). Thus, it is mandatory to perform a wide excision (if possible) as soon as when diagnostic work-up shows that an incomplete resection of the tumor has been performed. The same strategy should be applied in case of recurrence after a wide excision. This strategy avoids to perform a more extended resection and/or to use higher dose of radiotherapy in case of fast tumor growth.

Beatrice Aramini: The appropriate management strategy after UE is challenging and minimal guidance is provided in the existing literature. Management decisions may be impacted by a number of factors including, but not limited to, an analysis of preoperative imaging, histology, margin status, method of excision, surgeon experience, and sarcoma location. Management options include (I) observation, (II) wide re-excision alone, (III) preoperative radiation (RT) followed by wide re-excision, (IV) wide re-excision followed by adjuvant RT, and (V) RT alone. Due to the plethora of treatment options and number of complex, interrelated variables that may impact outcomes, treatment recommendations should evolve and emerge from the discussion by a team of experts at a multidisciplinary tumor board. Regardless of additional treatments, disease control after UE is compromised, with inferior outcomes, compared to patients who undergo PE by a sarcoma specialist. The optimal management of patients who undergo UE for their STS remains to be determined.

\section{Cecilia Pompili: /.}

Chaozong Liu: Again, this depends on the size, grade and health conditions of the patients.

Fabio Davoli: If unplanned it is not necessary, because you don't know the histopathologic nature. However, it should be a very rare situation, because of the high number of pre-operative exams that this kind of patients generally undergo. After accurate staging a wider excision has to be performed within one month after the first surgery.

Hans Van Veer: As soon as possible but not later than 4 weeks. Performing the secondary resection later will make the surgery more difficult as inflammation has set in the resected area.

Hiroaki Kuroda: /.

Ilhan Inci: Difficult situation. Completion of the incomplete resection should be planned 4 weeks after the first surgery. In this time period the surgical plans should be performed and most important the patient should have to recover for the next procedure.

Inderpal S. Sarkaria: /.

Ricciardi Sara: Each case should be discussed in the MDT meeting. The possibility to perform a new operation to obtain wide resection margin should be considered in relation to histology, ki67 (if applicable), clinical characteristic of patients, comorbidities etc. If indicated, the new surgery should be carried out in 30/40 days after the previous surgery.

Servet Bölükbas: Within 2-4 weeks.

Question 6: Due to the low incidence of chest wall sarcoma and lack of high-level clinical evidence, there is no TNM staging of chest wall sarcoma up to now. Currently, only the 8th edition TNM staging criteria of bone tumors (trunk, extremities, skull and maxillofacial) and soft tissue sarcomas (trunk and extremities) can be referenced according to the postoperative pathological diagnosis. Are the two staging system suitable for chest wall tumors?

Karel W. E. Hulsewé: The clinical value of staging systems 
for sarcomas are of limited value; a separate staging system for chest wall tumours may have scientific benefits, but clinical value remains to be determined.

Yvonne L. J. Vissers: No.

Erik R. de Loos: In my opinion, the aforementioned staging criteria are not sufficient for chest wall sarcoma. Despite the rather low incidence, a separate staging system for chest wall tumors would be very welcome for both clinical and scientific purposes.

Giuseppe Marulli: TNM staging bone and soft tissue sarcomas should not be properly used for chest wall sarcomas. The histologic types of bone and soft tissue sarcomas vary considerably and chest wall sarcoma has a histological and biological properties which are unique; therefore, the three factors of TNM are scarcely adaptable to all tissue types of sarcomas. In addition, because lymph node metastasis is extremely rare in bone soft tissue sarcomas and the $\mathrm{N}$ factor is rarely used, the stage would be determined by only two factors: T and M (3). They could be insufficient for a correct staging which should include also $\mathrm{G}$ for histologic grading, location of the primary sarcoma and its histological definition.

Alberto Sandri: Yes but not completely. An integration to the 8th edition TNM staging criteria of bone tumors and soft tissue sarcomas chest wall tumours with a specific chest wall TNM could be very interesting, although very challenging due to the rarity of the tumours and retrospective data of single institutions.

Andrea Bille: Yes, but an internation laregistry on chest wall sarcoma may help to attempt to create a specific TNM for chest wall sarcoma

Jin Yong Jeong: I have used the current staging system, although I believe that the current TNM staging criteria for bone tumors and soft tissue sarcomas are not suitable for chest wall sarcomas. I believe that special staging criteria for thoracic sarcoma are required in the future, and lymphadenectomy should be performed in the visible area or the adjacent mediastinal area.

Luca Ampollini: There is no an optimal staging system for chest wall sarcoma. Considering the spectrum of possible local and distant relapse, mostly due to the tumor biology, I think it will be tough to find an effective staging system and method to better frame these tumors. It might be obvious, but I would nevertheless like to point out that pathologists with extensive experience on chest wall sarcomas are undoubtedly best suited to understand and explain the most prominent features that characterized the histological aspects of these rare tumors.
Masatsugu Hamaji: Currently we have no answer. TNM staging of chest wall tumor should be attempted as clinical research.

Yuichiro Ueda: In my opinion, the two staging systems are not suitable for chest wall sarcomas. We should recognize that chest wall sarcoma is different from bone tumors and soft tissue sarcomas in other areas.

Zsolt Sziklavari: The 8th edition TNM staging criteria of soft tissue sarcomas (trunk and extremities) may be the most suitable staging system for chest wall tumors. However this system does not address special issues of chest wall tumors like the involvement of the lung for example. Due to the low incidence of chest wall sarcoma and the associated lack of high-level clinical evidence like already mentioned in the question there may be no better alternative up to now or in the near future because of the long timespan needed to capture the necessary data to provide a better staging system. Setting up an international database would be desirable.

Jacopo Vannucci: Staging system is based on data (collected and analyzed). The staging system can take inspiration from other tumors but it remains the final results of quality of the available data. Whether the staging system can follow the other bone tumors system cannot be supported or excluded but biology of these tumors are very different and so this eventuality appears unsound.

Alfonso Fiorelli: Surgical margin, size of the tumor, site of tumor, age of the patients, and tumor grade are all prognostic survival factors for chest wall sarcoma. Based on these evidences, the 8th edition TNM staging criteria of bone tumors (trunk, extremities, skull and maxillofacial) and soft tissue sarcomas is not fully suitable for chest wall tumors. In the future is mandatory to better define the exact localization of chest wall tumor and the depth. In fact, the chest wall is not a clearly defined anatomic site of origin, and it is generically defined as "trunk". Additionally, there is no clear differentiation based on the depth of infiltration of chest wall (superficial sarcoma or deep sarcoma). All these factors should better define in future classification as they are associated with different prognosis.

Beatrice Aramini: Soft tissue sarcomas of the head and neck (STSHN) usually present smaller than sarcomas of other sites, but carry a disproportionate risk of local recurrence. Up to $70 \%$ of tumors are less than $5 \mathrm{~cm}$ at presentation, and therefore classified together as T1. Given the rarity of STSHN, there is a paucity of data to guide progress in their classification.

Moreover, the majority of publications only report tumor size as less than or greater than $5 \mathrm{~cm}$, presumably based on 
conventions of the TNM system that remained unchanged for 40 years, thereby affecting progress of STSHN classification. This formed the impetus for change in the 8th edition in two key ways: (I) several soft tissue sarcoma site based changes occurred including STSHN now having its own system; and (II) primary tumor size cut-offs of 2 and $4 \mathrm{~cm}$ used in STSHN now reflect sizes that head and neck specialists commonly encounter in their practice. This update was pragmatic in modifying the TNM from a system with a T category not serving STSHN and which was originally based on sarcoma data from non-head and neck anatomic sites. The background to this change is outlined which provides a framework in which data can be reported to generate evidence for future staging modifications.

Cecilia Pompili: /.

Chaozong Liu: Not sure about this. Happy to know this should other colleagues have comments on it.

Fabio Davoli: No they aren't. Chest Wall Sarcomas are extremely rare tumors and classical TNM staging for bone tumors is not applicable in my opinion.

Hans Van Veer: TNM soft tissue sarcoma: probably yes.

TNM bone: Probably to few discrimination, as ribs are very small bones and before long, they will break out/though the cortices (same is valid for sternal tumours: thin bine of max $12-15 \mathrm{~mm}$ in comparison to e.g., a femur or tibia?).

Hiroaki Kuroda: /.

Ilhan Inci: To have one staging system is, I think, better than, to have no staging system. But it should be adopted or created newly in the international community.

Inderpal S. Sarkaria: /.

Ricciardi Sara: I think that a dedicated TNM staging for chest wall tumors should be achieved.

Servet Bölükbas: No. We need a TNM staging system for chest wall sarcoma.

Question 7: Traditionally, chest wall defects larger than $5 \mathrm{~cm}$ in maximum diameter should be reconstructed with rigid implants to prevent chest wall floating, paradoxical breathing, and/or respiratory failure. However, the above views are mainly based on the surgical experience and consensus of clinicians, and there is still a lack of high-level clinical evidence to confirm them. Is it necessary to use rigid implants for chest wall reconstruction once the maximum diameter of chest wall defect exceeds $5 \mathrm{~cm}$ in both adolescents and adults? What are your most common chest wall reconstruction materials?

Karel W. E. Hulsewé: Depending on the site of resection reconstruction is indicated. At the level of the scapular tip reconstruction of relatively smaller effects is indicated to prevent the scapula from slipping into the thoracic cavity. Most commonly a combination of plates and a polypropylene mesh is used in our clinic.

Yvonne L. J. Vissers: Matrix rib plates covered with polypropylene mesh, or polypropylene mesh only for the area at the level of the scapula.

Erik R. de Loos: The indication for chest wall reconstruction is predominantly determined by the anatomical proportions at the resection site, e.g., retroscapular defects resulting in intrathoracic displacement of the scapula or disturbed scapulothoracic movements should be reconstructed. Ideally, a combination of chest specific implants (e.g., MatrixRIB) and non-absorbable meshes (e.g., polypropylene) should be used.

Giuseppe Marulli: Usually, defects smaller than $5 \mathrm{~cm}$ in size in any location and those up to $10 \mathrm{~cm}$ in size posteriorly are not eligible for reconstruction, while larger defects, anterior defects or defects in proximity to the tip of the scapula must be reconstructed. The aim of reconstruction is to enable effective ventilation, avoid deformity and allow for adequate protection of internal organs; the use of a rigid prosthetic is thought to achieve all these goals. They have, however, also been associated with a risk of dislocation/ dislodgement, chronic periprosthetic infection and chronic pain (87-89).

Rigid prosthesis include plates of methacrylate, silicone, titanium and cyanoacrylate meshes. Methylmethacrylate plates provide excellent chest-wall stability and a low risk of respiratory complications, but it is associated with a great number of wound complications; further, fracture, tilting or extrusion of the implant are reported $(47,90)$. Titanium bars and rib clip are useful after a total sternectomy or after a wide antero-lateral chest-wall resection in a pediatric patient because of their flexibility so that the surgeon can model the reconstruction to the specific shape of the chest and of the defect.

Usually, a rigid prosthesis is beneficial although there is also evidence to suggest that less rigid biological meshes/ bioabsorbable plates provide better results in young, growing children $(51,91)$.

As non-rigid prosthetic materials, the most commonly used are Prolene or Marlex mesh and PTFE.

When the sternum is involved in the resection, there may be two subsets:

(I) The case of subtotal sternectomy, when a small part of the manubrium (with the sterno-clavicular 
joint) or a part of the lower sternal body are conserved, a rigid reconstruction is not necessary; further, these defects can be replaced by non-rigid prostheses;

(II) The case of large anterolateral chest-wall defects or in the case of a complete sternectomy, a reconstruction with rigid material is often mandatory to restore chest-wall stability and to maintain the geometry of the thoracic cage.

In the last forty years many materials have been proposed for the sternal replacement, ranging from soft to rigid materials. In recent years, the use of auto and allograft has been successfully adopted for sternal replacement. Its advantages include:

(I) To provide a rigid structure and a complete covering of the defect of the chest wall;

(II) Its capability of integration with the host patient's living tissue, thanks to their osteoconductive and osteoinductive capacities by acting as a scaffold for the genesis of new bone;

(III) To reduce the risk of infection and of immunologic reaction (92).

Alberto Sandri: Most surgeons agree that defects $>5 \mathrm{~cm}$ in diameter or including $>4$ ribs should be reconstructed due to cited above risks. In adults rigid implants do not represent an issue but they may in adolescents, however data is scare. My personal experience in adolescent chest reconstruction is small. Conceptually, in adolescents, there are some promising results with bioabsorbable materials for chest wall repair along with meshes to be preferred to any rigid implants, which should be avoided unless deemed necessary.

The ideal prosthetic material was defined by le Roux in early 80's (93): (I) rigidity to abolish paradoxical movement; (II) inertness to allow in-growth of fibrous tissue and decrease the likelihood of infection; (III) malleability to fashion to the appropriate shape at the time of operation; and (IV) radiolucency to create an anatomic reference to do a better follow up and identify a possible local neoplastic relapse.

Materials used for reconstructions:

Polypropylene, polyester, polytetrafluoroethylene PTFE (GORE-TEX); Vycril mesh,; Methyl Methacrylate meshes; biologic meshes such as XCM biologic ${ }^{\circledR}$ Tissue Matrix; bovine pericardium prosthesis; titanium meshes (MDF Medica); titanium plates (MatrixRIB; STRATOS system); Ley aluminium prostheses (for sternum); Watanabe et al. reported on the use of a sternal ceramic prosthesis constituted of hydroxyapatite and tricalcium phosphate (Ceratite) creating a customised prosthetic bone tailored to the anterior thoracic wall defects with slots and holes in the Ceratite prosthesis as fasteners; allografts and homografts, recovered from cadaveric donors $(94,95)$.

Andrea Bille: If the defect is behind the scapula you need a mesh but not rigid implants, in the other areas you should use a rigid implants or thick biological mesh.

Jin Yong Jeong: Although definitive guidelines for chest wall reconstruction have not been established, reconstruction of large defects after chest wall resection is necessary to protect organs within the thorax and to restore physiological chest wall movement. Titanium has high corrosion resistance, low specific gravity, good wear resistance, biological inertness and high biocompatibility. Therefore, I usually use a sandwich reconstruction technique using a titanium plate and a biological mesh because it can be safely used with a secondary prosthesis due to the nature of the biological mesh.

Luca Ampollini: When we talk about adolescents, we should keep in mind that rigid materials might impact on the physiological growth and expansion of the chest wall. So in these cases, a long term therapeutic strategy should be considered. A second surgical revision might be required in some patients. On the other hand, in adults, rigid implants should be always considered, depending on the site and surgeon's preference and attitude.

Synthetic meshes of polypropylene, polytetrafluoroethylene (PTFE), polyglactin 910, or polypropylene mesh-methyl methacrylate composite have been used to achieve chest wall stability. In our center we mainly use PTFE. Even considering the rarity of these tumors, over the years we have gained a nice experience by utilizing this material.

Although PTFE is more resistant to infection and less prone to adhesions than other synthetics, its incorporation into surrounding tissue may be inferior to that of woven or knitted materials. For a few years now, biomaterials have been introduced for abdominal wall reconstruction, particularly in the setting of overtly or potentially contaminated fields, with promising results. The use of bovine pericardium patch and polylactic acid (PLA) bars used solely or combined for reconstruction and stabilization of the thoracic skeleton have been reported as a valuable option in the management of patients with chest wall tumors.

Masatsugu Hamaji: Rigid fixation of even small resection of the anterior chest wall is preferable, on the basis of my animal experiment (96). On the other hand, metal materials 
for the anterior chest wall may not be associated with longterm fixation, which requires careful attention (97). My most common reconstruction material is polytetrafluoroethylene for the lateral chest wall.

Yuichiro Ueda: Chest wall reconstruction occurs most commonly when a surgical procedure involves the removal of multiple ribs or more than $5 \mathrm{~cm}$ of a rib since in such cases, thinning of the anterior wall and the posterior wall adjacent to the scapula angle is likely to lead to a deformity.

PTFE is a widely used reconstruction material in our country, but rigid implants such as titanium plates should be used for the wide chest wall defect. We developed a rib socket for rib fracture and are now seeking application for chest wall reconstruction.

Zsolt Sziklavari: For defects beneath the scapula, chest wall reconstruction is not required. For the rest we prefer synthetic prosthetic materials. We close three-ribswindows. These materials can be utilized to attain rib or sternal stability. Placing any of these materials under tension improves the rigidity of the prosthesis in all directions. Gore-Tex is cool but expensive. Big and full-thickness defects should be by a plastic surgeon shown. We do not use routinely neo-ribs.

Jacopo Vannucci: I believe that the "area" of the defect is not a "good" parameter. A series of parameters better helps in the decision-making process. Positions, anatomical condition, functional condition et cetera play a role. There is no perfect material for reconstruction, so I use different materials. For small defects, I prefer meshes; for wide defects, I believe titanium gives more stability. Biomimesis and functional stability is the "light at the end of the tunnel". The better these two parameters, the better the outcome. I am afraid that experience is still more important than clinical standards.

Alfonso Fiorelli: The decision for reconstruction of chest wall extent should be done not only based on the diameter of the defect but also on the site of the defect. For example, defects larger than $5 \mathrm{~cm}$ in diameter may not require any reconstruction whether localized posteriorly under scapula above the fourth rib. In these cases, the skeletal component can be ignored and the defect closed with only soft tissue. Conversely, small defect less than $5 \mathrm{~cm}$ in diameter could need a reconstruction if they are located in anterior or lateral chest wall as the high risk of chest wall floating, paradoxical breathing, and/or respiratory failure. How to reconstruct the chest wall defect depends on the available materials and the surgeon's experience. The use of synthetic mesh such as Marlex, Prolene or polytetrafluoroethylene either alone or reinforced with methyl methacrylate and local muscle flaps (i.e., latissimus dorsi, pectoralis major, serratus anterior, rectus abdominis, external oblique and trapezius) is a safe, effective one-stage surgical procedure for reconstruction a variety of major chest wall defects. The use of titanium bars fixed on remaining rib segments is an alternative to the above reported strategies.

Beatrice Aramini: For example, the sternum is the pivotal part of the chest wall, connecting clavicles and ribs on both sides. When the defect exceeds $5 \times 5 \mathrm{~cm}^{2}$ after sternal resection, reconstruction is justified to restore the bony chest wall. Titanium plates or meshes, Sandwich patches, auto- or allografts of bone materials, and 3D printing prostheses are often used for reconstruction. At present, there is no such ideal prosthesis that meets the requirement for sternal reconstruction, such as compatibility, mechanical strength, and tailorability.

Large soft-tissue defects after tumor resection can be covered by local, pedicled, or free flaps. In cases of large full-thickness defects, flaps can be combined with polypropylene mesh to improve chest wall stability and to maintain pulmonary function.

Cecilia Pompili: /.

Chaozong Liu: Additively manufactured PEEK and Titanium implants could be used for reconstruction.

Fabio Davoli: The cut-off of 5 centimeters is good to determine. If the defect is larger, and/or it belongs to a critical area for the dynamic of the thoracic cage, you have to reconstruct. An ideal prosthetic material is elastic, resistant, biocompatible and adaptable to the surface to be replaced. I usually replace chest wall defects with a GoreTex mesh. An alternative choice could be represented by a fascia lata graft.

Hans Van Veer: Indeed, the most important reason to reconstruct a chest wall defect is to prevent lung herniation and to permit normal physiologic movements of the chest wall. Respecting this, normal breathing should not be hindered, and as such complications like pneumonia could be prevented. Bearing this in mind, the defect needs to be reconstructed especially on the anterior and lateral chest wall. The more anterior, the more 'play' one will find during respiratory movement at the level of the rib, leading to a negative impact on respiratory movement of the chest wall. As such: a strong plea for semirigid fixation $+/-$ sheath (e.g., Gorete) to prevent lung herniation in between the material and the ribs. All removed levels should be bridged as to restore the flexible rigidity of the chest wall.

When occurring at the posterolateral chest wall and 
behind the scapula, the scapula will prevent lung herniation. Moreover, the closer the defect is towards the vertebral column, the less movement is expected in the ray of the rib. However, when the defect is at such a location that the scapula tip can get in during shoulder movement, reconstruction with semi-rigid material should be performed as inward herniation of the scapula tip will lead to blockage and pain.

I use as rib reconstruction material titanium plates (biomet/Synthes) and goretex $2 \mathrm{~mm}$ mesh for the prevention of lung- or mediastinal organ herniation. Goretex $1 \mathrm{~mm}$ for pericardial reconstruction. The titanium plates are in my mind rigid enough but with some flexibility.

I do not use PEEK; I do not use the clips around the ribs (stracos) as for applying these instruments, I feel the soft tissues have to hampered once more, besides the extent of the resection.

I do not have experience with $3 \mathrm{D}$ printed material.

I do not like methylmetacrylate cement plates, as these are too rigid and thus non-physiologic, besides very frequent occurring seromata.

Hiroaki Kuroda: /.

Ilhan Inc: In my current practice I use, muscle flap (with the help of plastic surgeon) and synthetic material (GoreTex or Prolene Mesh) sometimes methylmetacrylate (bone cement). I also make ribs from bone cement to prevent the flailing of the chest defect.

Inderpal S. Sarkaria: /.

Ricciardi Sara: In my opinion the rigid implants allow a more physiological reconstruction and also guarantee a better aesthetic result. In my practice I usually applied titanium bar + Gore-Tex mesh.

Servet Bölükbas: In my experience, rigid reconstruction is recommended in the anterior chest wall in order to have a protection of the chest organs. We should differ between reconstruction of the anterior and posterior chest wall. Not every defect of $5 \mathrm{~cm}$ needs to be reconstructed rigidly. In case of rigid reconstruction, I prefer polymethylmethacrylate.

\section{Question 8: It is a rare clinical disease of lung cancer invading the chest wall, accounting for about $5 \%$ of all cases. A number of retrospective clinical studies have shown that patients with lung cancer invading the chest wall can benefit from surgery, especially ones without lymph node metastasis. For the NSCLC invading the chest wall $\left(T_{3-4} N_{0-1} M_{0}\right)$, is wide excision necessary for these patients?}

Karel W. E. Hulsewé: Yes, radical resection is indicated with a wide margin in order to obtain a R0 resection. We aim for a margin of approximately $2 \mathrm{~cm}$.

Yvonne L. J. Vissers: Yes.

Erik R. de Loos: Yes, with a resection margin of 2 centimeters. According to both Dutch and European guidelines, adjuvant chemotherapy is indicated for $\mathrm{pT}_{3-4} \mathrm{~N}_{0-1}$ tumors. In case of $\mathrm{R} 1$ resections, adjuvant radiotherapy is indicated as well.

Giuseppe Marulli: The complete surgical resection via lobectomy with en bloc chest wall resection represents the gold standard for curative intent, with a 40-50\% expected 5 -year survival when a complete resection is achieved (R0) and when there is no nodal involvement (N0).

The adjunct of chest wall resection and reconstruction carries a not negligible increase in terms of morbidity and mortality (up to $9 \%$ in recent series) if compared to conventional standard lung resections (2). R0 resection and lymph node invasion are the major prognostic factors for lung cancer invading the chest wall.

Alberto Sandri: Yes, it is if the patient is fit enough. However, such decisions should be discussed at MDTs and with the patient.

Andrea Bille: Yes it is.

Jin Yong Jeong: For lung cancer with the chest wall invasion, surgery including extensive chest wall resection is considered unless it is N2 disease confirmed by mediastinoscopy or EBUS-TBNA examination. Scarnecchia et al. (67) performed major lung resection en bloc with the chest wall in 54 patients with NSCLC invading the chest wall. A retrospective analysis of these data concluded that $\mathrm{N} 0$ status and free resection margin are the major oncological prognostic factors for these patients. I have performed surgery on a patient with lung cancer invading the chest wall if $\mathrm{N} 1$ disease was not confirmed before surgery and the patient's condition was operable. And I believe that extensive resection is necessary for free resection margin.

Luca Ampollini: It has been shown that a realistic chance to cure locally advanced tumors invading the chest wall (CW) is a surgical resection, consisting in the excision of the primary lung cancer along with the involved CW (sometimes an "en-bloc" resection) and an accurate lymphnode dissection. The prognosis mainly depends on the completeness of resection (R0) and possible lymph-node involvement. A multidisciplinary approach is even more important in these patients' subgroup. Depending on the surgical team expertise, a plastic surgeon (for flap harvesting or microvascular anastomosis), a neurosurgeon (in case of 
vertebral body invasion) might be involved for planning the optimal treatment strategy.

Masatsugu Hamaji: Not wide excision but about $2 \mathrm{~cm}$ margin appears appropriate for chest wall resection for NSCLC.

Yuichiro Ueda: Patients with lung cancer invading the chest wall might benefit from surgery. However, distant metastasis often occurs during the observation period. Adjuvant chemotherapy should be recommended.

Zsolt Sziklavari: The median number of resected ribs in Coburg in the last five years was three (98-102). I think also, that the involved ribs should be transected macroscopic clean and $2 \mathrm{~cm}$ beyond the margin of gross involvement. A frozen section confirmation is obligatory. I am for R0 here.

I have used the following sources and books as references. $\mathrm{R} 0$ means for us macroscopic $\mathrm{R} 0$ resection $+2 \mathrm{~cm}$ safety distance. R0-max means macroscopic R0 resection $+4 \mathrm{~cm}$ safety distance.

Jacopo Vannucci: Lung cancer invading the chest wall is a particular condition. Resection margin is important but far less important than for sarcomas. Considering the survival reported from a series of reports, the best approach is lobectomy with en bloc chest wall resection. Width and clear margin are more than important. A well-performed resection gives a chance of cure while survival drops after $\mathrm{R} 1$ resections.

Alfonso Fiorelli: Survival of patients with lung cancer invading the chest wall mainly depends on lymph node involvement. In some patients with $\mathrm{T}_{3} \mathrm{~N}_{0}$ disease, 5 -year survival in excess of $50 \%$ can be achieved after surgery. In patients with $\mathrm{N} 1$ or N2 disease the role of surgery is controversial. The 5 -year survival is $20-25 \%$ in $\mathrm{N} 1$ and below $10 \%$ in $\mathrm{N} 2$ patients. Thus, surgery is indicated as the primary treatment modality in $\mathrm{T}_{3-4} \mathrm{~N}_{0-1}$ NSCLC. In the presence of N2 involvement, neoadjuvant chemotherapy followed by surgical re-evaluation is the most valuable option. Other prognostic survival factors are the margin of resection and the depth of infiltration. R1 and R2 resection may significantly impair the survival and these subsets of patients should benefit from adjuvant radiotherapy. Tumor invasion limited to parietal pleura may be treated with extra-pleural dissection alone rather than chest wall resection. This strategy limits the extent of the resection without jeopardizing the oncological safety of the procedure.

Beatrice Aramini: Whether adjuvant radiotherapy is needed in chest wall $\mathrm{pT}_{3}$ NSCLC is still an open issue. We did not observe any difference in terms of local relapse and overall survival in stage IIB patients with regard to the administration of postoperative radiotherapy. In contrast, adjuvant radiotherapy increased survival in stage IIIA patients. It may be hypothesized that N0 patients having undergone a R0 resection did not need any adjuvant radiotherapy to achieve a suitable local control of the disease. Conversely, besides the bias due to the frequent adjunction of chemotherapy in stage IIIA patients, it may be speculated that mediastinal radiotherapy improved the local control of the disease in $\mathrm{N}+$ patients. However, our study, as well as most publications, sins by the absence of a uniform protocol employed in those patients.

To conclude, the present results highlight several particular features of chest wall $\mathrm{pT}_{3}$ NSCLC patients. The disastrous impact of lymph node metastases on survival leads to questions of whether surgery is of any benefit in this subset of patients. The best surgical candidates are likely to be those with a N0 disease. In that way, en-bloc resection is strongly suggested to be the standard of surgical care, and adjuvant radiotherapy does not seem to be necessary if a complete resection has been achieved. For huge tumors, this report suggests that the role of perioperative chemotherapy needs further evaluation.

Cecilia Pompili: /.

Chaozong Liu: I would like to recommend wide excision for reducing recurrence.

Fabio Davoli: Absolutely yes.

If you deal with a locally advanced NSCLS invading the chest wall, R0 resection with reasonable surgical margins can have a significant impact on long time survival, especially for N0 patients. Anyway, every clinical decision must be taken under a multidisciplinary team supervision.

Hans Van Veer: Yes; I agree with the proposed statement as concluded in statement 6 .

Hiroaki Kuroda: /.

Ilhan Inci: I think, in lung cancer surgery, complete resection is important, and wide resection should be part of it.

Inderpal S. Sarkaria: /.

Ricciardi Sara: Yes, R0 resection should be obtained.

Servet Bölükbas: Yes, I would plea for $4 \mathrm{~cm}$. Not rarely, discontinuing tumor spread can be found at the resection margins. During surgery, frozen section is not possible to examine the osseus resection margins. More safety margin should translate in more complete resections.

\section{Acknowledgments}

Funding: The work was supported by National Key R\&D 
Program of China (2018YFE0207900), Top-notch project of medical science and technology for youth cultivation of the army(18QNP028), Clinical Development Innovation Fund of Air Force Medical University (2021XB024), Lingyun Program of Air Force Medical University (2019cyjhwl), Zhufeng program of Air Force Medical University (2019rcfcyxl), Key R\&D Program of Guangdong Province (2018B090906001), and the National Natural Science Foundation of China (51835010).

\section{Footnote}

Conflicts of Interest: All authors have completed the ICMJE uniform disclosure form (available at https://dx.doi. org/10.21037/tlcr-21-935). Dr. ISS reports that he had received grants from On Target Laboratories; payment or honoraria for lectures, presentations, speakers bureaus, manuscript writing or educational events form Intuitive Surgical, Inc., Cambridge Medical Robotics and Auris Medical. Dr. XY serves as an unpaid editorial board member of Translational Lung Cancer Research from July 2021 to June 2023. The other authors have no conflicts of interest to declare.

Ethical Statement: The authors are accountable for all aspects of the work in ensuring that questions related to the accuracy or integrity of any part of the work are appropriately investigated and resolved.

Open Access Statement: This is an Open Access article distributed in accordance with the Creative Commons Attribution-NonCommercial-NoDerivs 4.0 International License (CC BY-NC-ND 4.0), which permits the noncommercial replication and distribution of the article with the strict proviso that no changes or edits are made and the original work is properly cited (including links to both the formal publication through the relevant DOI and the license). See: https://creativecommons.org/ licenses/by-nc-nd/4.0/.

\section{References}

1. Smith SE, Keshavjee S. Primary chest wall tumors. Thorac Surg Clin 2010;20:495-507.

2. Fletcher CDM, Bridge JA, Hogendoorn PCW, et al. WHO classification of tumors of soft tissue and bone, 4th Edition.Lyon:LARC Press, 2013:244-7.

3. Tanaka K, Ozaki T. New TNM classification (AJCC eighth edition) of bone and soft tissue sarcomas: JCOG Bone and Soft Tissue Tumor Study Group. Jpn J Clin Oncol 2019;49:103-7.

4. NCCN, The NCCN Soft Tissue Sarcoma Clinical Practice Guidelines in Oncology (version 2. 2020). Fort Washington: NCCN, 2020.

5. NCCN, The NCCN Bone Cancer Clinical Practice Guidelines in Oncology (version 1. 2020). Fort Washington: NCCN, 2020.

6. Thoracic Surgeons Branch of Chinese Medical Doctor Association, Chinese expert consensus on chest wall tumor resection and chest wall reconstruction surgery (2018 edition). Chinese Journal of Thoracic and Cardiovascular Surgery, 2018.

7. Kabiri EH, Al Aziz S, El Maslout A, et al. Desmoid tumors of the chest wall. Eur J Cardiothorac Surg 2001;19:580-3.

8. Eastley NC, Hennig IM, Esler CP, et al. Nationwide trends in the current management of desmoid (aggressive) fibromatosis. Clin Oncol (R Coll Radiol) 2015;27:362-8.

9. Kuwahara H, Salo J, Nevala R, et al. Single-Institution, Multidisciplinary Experience of Soft Tissue Sarcomas in the Chest Wall. Ann Plast Surg 2019;83:82-8.

10. Kachroo P, Pak PS, Sandha HS, et al. Single-institution, multidisciplinary experience with surgical resection of primary chest wall sarcomas. J Thorac Oncol 2012;7:552-8.

11. Mátrai Z, Tóth L, Szentirmay Z, et al. Sporadic desmoid tumors of the chest: long-term follow-up of 28 multimodally treated patients. Eur J Cardiothorac Surg 2011;40:1170-6.

12. Abbas AE, Deschamps C, Cassivi SD, et al. Chest-wall desmoid tumors: results of surgical intervention. Ann Thorac Surg 2004;78:1219-23; discussion 1219-23.

13. Gronchi A, Casali PG, Mariani L, et al. Quality of surgery and outcome in extra-abdominal aggressive fibromatosis: a series of patients surgically treated at a single institution. J Clin Oncol 2003;21:1390-7.

14. Peng PD, Hyder O, Mavros MN, et al. Management and recurrence patterns of desmoids tumors: a multiinstitutional analysis of 211 patients. Ann Surg Oncol 2012;19:4036-42.

15. Mullen JT, Delaney TF, Kobayashi WK, et al. Desmoid tumor: analysis of prognostic factors and outcomes in a surgical series. Ann Surg Oncol 2012;19:4028-35.

16. NCCN, The NCCN Clinical Practice Guidelines in Oncology: soft tissue sarcoma (version 4. 2021). Fort Washington: NCCN, 2021. 
17. Reed V, Shah J, Medeiros LJ, et al. Solitary plasmacytomas: outcome and prognostic factors after definitive radiation therapy. Cancer 2011;117:4468-74.

18. Nakanishi K, Kashiwagi N, Hamada K, et al. Solitary plasmacytoma of the sternum detected incidentally by MR imaging of the cervical spine. Magn Reson Med Sci 2010;9:227-31.

19. Woodruff RK, Malpas JS, White FE. Solitary plasmacytoma. II: Solitary plasmacytoma of bone. Cancer 1979;43:2344-7.

20. Jin Yong Jeong, NCCN, The NCCN Clinical Practice Guidelines in Oncology:Multiple Myeloma (version 1. 2021). Fort Washington: NCCN, 2021.

21. Safi S, Op den Winkel J, Schnabel PA, et al. Extended resection of a plasmocytoma of bone and an amyloidoma of the chest wall. Ann Thorac Surg 2013;96:2223-5.

22. Jia R, Xue L, Liang H, et al. Surgery combined with radiotherapy for the treatment of solitary plasmacytoma of the rib: a case report and review of the literature. J Cardiothorac Surg 2015;10:125.

23. Sabanathan S, Shah R, Mearns AJ. Surgical treatment of primary malignant chest wall tumours. Eur J Cardiothorac Surg 1997;11:1011-6.

24. Pezzella AT, Fall SM, Pauling FW, et al. Solitary plasmacytoma of the sternum: surgical resection with longterm follow-up. Ann Thorac Surg 1989;48:859-62.

25. Martini N, Huvos AG, Burt ME, et al. Predictors of survival in malignant tumors of the sternum. J Thorac Cardiovasc Surg 1996;111:96-105; discussion 105-6.

26. Liu X, Huang LJ, Liu YJ, et al. A preliminary exploratory study of $3 \mathrm{D}$ printed polyether ether ketone thoracic rib implant in the surgical treatment of sternum plasmacytoma. Chinese J Thorac Cardiovasc Surg 2021;37:149.

27. Caers J, Paiva B, Zamagni E, et al. Diagnosis, treatment, and response assessment in solitary plasmacytoma: updated recommendations from a European Expert Panel. J Hematol Oncol 2018;11:10.

28. McMillan RR, Sima CS, Moraco NH, et al. Recurrence patterns after resection of soft tissue sarcomas of the chest wall. Ann Thorac Surg 2013;96:1223-8.

29. Tsukushi S, Nishida Y, Sugiura H, et al. Soft Tissue Sarcomas of the Chest Wall. J Thorac Oncol 2009;4:834-7.

30. van Geel AN, Wouters MW, Lans TE, et al. Chest wall resection for adult soft tissue sarcomas and chondrosarcomas: analysis of prognostic factors. World J Surg 2011;35:63-9.
31. Harati K, Kolbenschlag J, Bohm J, et al. Long-term outcomes of patients with soft tissue sarcoma of the chest wall: Analysis of the prognostic significance of microscopic margins. Oncol Lett 2018;15:2179-87.

32. Park I, Shin S, Kim HK, et al. Primary Chest Wall Sarcoma: Surgical Outcomes and Prognostic Factors. Korean J Thorac Cardiovasc Surg 2019;52:360-7.

33. King RM, Pairolero PC, Trastek VF, et al. Primary chest wall tumors: factors affecting survival. Ann Thorac Surg 1986;41:597-601.

34. Shewale JB, Mitchell KG, Nelson DB, et al. Predictors of survival after resection of primary sarcomas of the chest wall-A large, single-institution series. J Surg Oncol 2018;118:518-24.

35. Wouters MW, van Geel AN, Nieuwenhuis L, et al. Outcome after surgical resections of recurrent chest wall sarcomas. J Clin Oncol 2008;26:5113-8.

36. Walsh GL, Davis BM, Swisher SG, et al. A singleinstitutional, multidisciplinary approach to primary sarcomas involving the chest wall requiring full-thickness resections. J Thorac Cardiovasc Surg 2001;121:48-60.

37. Bhangu AA, Beard JA, Grimer RJ. Should Soft Tissue Sarcomas be Treated at a Specialist Centre? Sarcoma 2004;8:1-6.

38. Noria S, Davis A, Kandel R, et al. Residual disease following unplanned excision of soft-tissue sarcoma of an extremity. J Bone Joint Surg Am 1996;78:650-5.

39. Arai E, Nishida Y, Tsukushi S, et al. Clinical and treatment outcomes of planned and unplanned excisions of soft tissue sarcomas. Clin Orthop Relat Res 2010;468:3028-34.

40. Fiore M, Casali PG, Miceli R, et al. Prognostic effect of re-excision in adult soft tissue sarcoma of the extremity. Ann Surg Oncol 2006;13:110-7.

41. Chandrasekar CR, Wafa H, Grimer RJ, et al. The effect of an unplanned excision of a soft-tissue sarcoma on prognosis. J Bone Joint Surg Br 2008;90:203-8.

42. Chui CH, Spunt SL, Liu T, et al. Is re-excision in pediatric nonrhabdomyosarcoma soft tissue sarcoma necessary after an initial unplanned resection? J Pediatr Surg 2002;37:1424-9.

43. Zornig C, Peiper M, Schröder S. Re-excision of soft tissue sarcoma after inadequate initial operation. Br J Surg 1995;82:278-9.

44. Zagars GK, Ballo MT, Pisters PW, et al. Surgical margins and reresection in the management of patients with soft tissue sarcoma using conservative surgery and radiation therapy. Cancer 2003;97:2544-53. 
45. Guillou L, Coindre JM, Bonichon F, et al. Comparative study of the National Cancer Institute and French Federation of Cancer Centers Sarcoma Group grading systems in a population of 410 adult patients with soft tissue sarcoma. J Clin Oncol 1997;15:350-62.

46. Riad S, Griffin AM, Liberman B, et al. Lymph node metastasis in soft tissue sarcoma in an extremity. Clin Orthop Relat Res 2004;(426):129-34.

47. Weyant MJ, Bains MS, Venkatraman E, et al. Results of chest wall resection and reconstruction with and without rigid prosthesis. Ann Thorac Surg 2006;81:279-85.

48. Zhang Y, Li JZ, Hao YJ, et al. Sternal tumor resection and reconstruction with titanium mesh: a preliminary study. Orthop Surg 2015;7:155-60.

49. Saltsman JA, Danzer E, Hammond WJ, et al. Survival and Scoliosis Following Resection of Chest Wall Tumors in Children and Adolescents: A Single-center Retrospective Analysis. Ann Surg 2021;274:e167-73.

50. Lopez C, Correa A, Vaporciyan A, et al. Outcomes of chest wall resections in pediatric sarcoma patients. J Pediatr Surg 2017;52:109-14.

51. Lin SR, Kastenberg ZJ, Bruzoni M, et al. Chest wall reconstruction using implantable cross-linked porcine dermal collagen matrix (Permacol). J Pediatr Surg 2012;47:1472-5.

52. Glotzbecker MP, Gold M, Puder M, et al. Scoliosis after chest wall resection. J Child Orthop 2013;7:301-7.

53. Voss B, Bauernschmitt R, Will A, et al. Sternal reconstruction with titanium plates in complicated sternal dehiscence. Eur J Cardiothorac Surg 2008;34:139-45.

54. Berthet JP, Canaud L, D'Annoville T, et al. Titanium plates and Dualmesh: a modern combination for reconstructing very large chest wall defects. Ann Thorac Surg 2011;91:1709-16.

55. Girotti P, Leo F, Bravi F, et al. The "rib-like" technique for surgical treatment of sternal tumors: lessons learned from 101 consecutive cases. Ann Thorac Surg 2011;92:1208-15; discussion 1215-6.

56. Wang L, Cao T, Li X, et al. Three-dimensional printing titanium ribs for complex reconstruction after extensive posterolateral chest wall resection in lung cancer. J Thorac Cardiovasc Surg 2016;152:e5-7.

57. Wang L, Huang L, Li X, et al. Three-Dimensional Printing PEEK Implant: A Novel Choice for the Reconstruction of Chest Wall Defect. Ann Thorac Surg 2019;107:921-8.

58. Aranda JL, Jiménez MF, Rodríguez M, et al. Tridimensional titanium-printed custom-made prosthesis for sternocostal reconstruction. Eur J Cardiothorac Surg 2015;48:e92-4.

59. Moradiellos J, Amor S, Córdoba M, et al. Functional Chest Wall Reconstruction With a Biomechanical ThreeDimensionally Printed Implant. Ann Thorac Surg 2017;103:e389-91.

60. Simal I, García-Casillas MA, Cerdá JA, et al. ThreeDimensional Custom-Made Titanium Ribs for Reconstruction of a Large Chest Wall Defect. European J Pediatr Surg Rep 2016;4:26-30.

61. Vannucci J, Scarnecchia E, Potenza R, et al. Dynamic titanium prosthesis based on 3D-printed replica for chest wall resection and reconstruction. Transl Lung Cancer Res 2020;9:2027-32.

62. Facciolo F, Cardillo G, Lopergolo M, et al. Chest wall invasion in non-small cell lung carcinoma: a rationale for en bloc resection. J Thorac Cardiovasc Surg 2001;121:649-56.

63. Riquet M, Lang-Lazdunski L, Le PB, et al. Characteristics and prognosis of resected T3 non-small cell lung cancer. Ann Thorac Surg 2002;73:253-8.

64. Matsuoka H, Nishio W, Okada M, et al. Resection of chest wall invasion in patients with non-small cell lung cancer. Eur J Cardiothorac Surg 2004;26:1200-4.

65. Doddoli C, D'Journo B, Le Pimpec-Barthes F, et al. Lung cancer invading the chest wall: a plea for en-bloc resection but the need for new treatment strategies. Ann Thorac Surg 2005;80:2032-40.

66. Lin YT, Hsu PK, Hsu HS, et al. En bloc resection for lung cancer with chest wall invasion. J Chin Med Assoc 2006;69:157-61.

67. Scarnecchia E, Liparulo V, Capozzi R, et al. Chest wall resection and reconstruction for tumors: analysis of oncological and functional outcome. J Thorac Dis 2018;10:S1855-63.

68. Jones GD, Caso R, No JS, et al. Prognostic factors following complete resection of non-superior sulcus lung cancer invading the chest wall. Eur J Cardiothorac Surg 2020;58:78-85.

69. Magdeleinat P, Alifano M, Benbrahem C, et al. Surgical treatment of lung cancer invading the chest wall: results and prognostic factors. Ann Thorac Surg 2001;71:1094-9.

70. NCCN, The NCCN Non Small Cell Lung Cancer Clinical Practice Guidelines in Oncology (version 1. 2020). Fort Washington: NCCN, 2020.

71. Dashiell TG, Payne WS, Hepper NG, et al. Desmoid tumors of the chest wall. Chest 1978;74:157-62.

72. Ibrahim M, Sandogji H, Allam A. Huge intrathoracic 
desmoid tumor. Ann Thorac Med 2009;4:146-8.

73. Takeshima Y, Nakayori F, Nakano T, et al. Extraabdominal desmoid tumor presenting as an intrathoracic tumor: case report and literature review. Pathol Int 2001;51:824-8.

74. Meyerson SL, D'Amico TA. Intrathoracic desmoid tumor: brief report and review of literature. J Thorac Oncol 2008;3:656-9.

75. Desmoid Tumor Working Group. The management of desmoid tumours: A joint global consensus-based guideline approach for adult and paediatric patients. Eur J Cancer 2020;127:96-107.

76. Brodsky JT, Gordon MS, Hajdu SI, et al. Desmoid tumors of the chest wall. A locally recurrent problem. J Thorac Cardiovasc Surg 1992;104:900-3.

77. Abrão FC, Waisberg DR, Fernandez A, et al. Desmoid tumors of the chest wall: surgical challenges and possible risk factors. Clinics (Sao Paulo) 2011;66:705-8.

78. Lev D, Kotilingam D, Wei C, et al. Optimizing treatment of desmoid tumors. J Clin Oncol 2007;25:1785-91.

79. Suh YG, Suh CO, Kim JS, et al. Radiotherapy for solitary plasmacytoma of bone and soft tissue: outcomes and prognostic factors. Ann Hematol 2012;91:1785-93.

80. Sasaki R, Yasuda K, Abe E, et al. Multi-institutional analysis of solitary extramedullary plasmacytoma of the head and neck treated with curative radiotherapy. Int J Radiat Oncol Biol Phys 2012;82:626-34.

81. Burt A, Berriochoa J, Korpak A, et al. Treatment of chest wall sarcomas: a single-institution experience over 20 years. Am J Clin Oncol 2015;38:80-6.

82. Kucharczuk JC. Chest wall sarcomas and induction therapy. Thorac Surg Clin 2012;22:77-81, vii.

83. Sacchetti F, Alsina AC, Morganti R, et al. Re-excision after unplanned excision of soft tissue sarcoma: A systematic review and metanalysis. The rationale of systematic reexcision. J Orthop 2021;25:244-51.

84. Kang J, Xu M, Wang B, et al. Wide resection of soft tissue sarcomas after unplanned primary procedures: A long-term follow-up study. Medicine (Baltimore) 2020;99:e19067.

85. Decanter G, Stoeckle E, Honore C, et al. Watch and Wait Approach for Re-excision After Unplanned Yet Macroscopically Complete Excision of Extremity and Superficial Truncal Soft Tissue Sarcoma is Safe and Does Not Affect Metastatic Risk or Amputation Rate. Ann Surg Oncol 2019;26:3526-34.

86. Bonvalot S, Levy A, Terrier P, et al. Primary extremity soft tissue sarcomas: does local control impact survival? Ann
Surg Oncol 2017;24:194-201.

87. Walton JM, Bass J, Sambey E, et al. Use of human dura in pediatric chest wall reconstruction after tumor resection. J Pediatr Surg 1994;29:1189-91.

88. La Quaglia MP. Chest wall tumors in childhood and adolescence. Semin Pediatr Surg 2008;17:173-80.

89. Oliveira C, Zamakhshary M, Alfadda T, et al. An innovative method of pediatric chest wall reconstruction using Surgisis and swinging rib technique. J Pediatr Surg 2012;47:867-73.

90. Mansour KA, Thourani VH, Losken A, et al. Chest wall resection and reconstruction: a 25-year experience. Ann Thorac Surg 2002,73:1720-5.

91. Tuggle DW, Mantor PC, Foley DS, et al. Using a bioabsorbable copolymer plate for chest wall reconstruction. J Pediatr Surg 2004;39:626-8.

92. Marulli G, De Iaco G, Ferrigno P, et al. Sternochondral replacement: use of cadaveric allograft for the reconstruction of anterior chest wall. J Thorac Dis 2020;12:3-9.

93. le Roux BT, Shama DM. Resection of tumors of the chest wall. Curr Probl Surg 1983;20:345-86.

94. Stella F, Dell'Amore A, Dolci G, et al. Allogenic sternal transplant after sternectomy for metastasis of ovarian carcinoma. Ann Thorac Surg 2012;93:e71-2.

95. Watanabe A, Watanabe T, Obama T, et al. New material for reconstruction of the anterior chest wall, including the sternum. J Thorac Cardiovasc Surg 2003;126:1212-4.

96. Hamaji M, Kojima F, Koyasu S, et al. Rigid and bioabsorbable material for anterior chest wall reconstruction in a canine model. Interact Cardiovasc Thorac Surg 2015;20:322-8.

97. Hamaji M. Still a Long Way to Go for Anterior or Extensive Chest Wall Reconstruction. Ann Thorac Surg 2016;101:410.

98. Soft Tissue and Bone Tumours, WHO Classification of Tumours, 5th Edition, Volume 3 Chapter 1, 2020 (WHO Editorial Board WHO Classification of Tumours: Soft Tissue and Bone Tumours, ISBN: 97892-8324502-5.

99. Expertise Thoraxchirurgie. Michael Rolf Müller, Stefan B. Watzka (Herausgeber), 2015, 1. Auflage. Thieme ISBN: 978-3-13-175031-0.

100. German Guideline; S3-Leitlinie. Prävention, Diagnostik, Therapie und Nachsorge des Lungenkarzinoms. 2018.

101.Pearson's Thoracic and Esophageal Surgery. Edited by G. Alexander Patterson et al. Expert Consult: Online and 
Print, 2-Volume Set. 3. Edition, 2008. ISBN-13: 978-0443-06861-4.

102. General thoracic surgery. Edited by Thomas W. Shields et al. Lippincott Williams \& Wilkins, a Wolters Kluwer business. 7th. Edition. 2005. ISBN: 978-0-7817-7982-1.

(English Language Editor: A. Kassem)

Cite this article as: Wang L, Yan X, Zhao J, Chen C, Chen C, Chen J, Chen KN, Cao T, Chen MW, Duan H, Fan J, Fu J, Gao S, Guo H, Guo S, Guo W, Han Y, Jiang GN, Jiang H, Jiao WJ, Kang M, Leng X, Li HC, Li J, Li J, Li SM, Li S, Li Z, Li Z, Liang C, Mao NQ, Mei H, Sun D, Wang D, Wang L, Wang Q, Wang S, Wang T, Liu L, Xiao G, Xu S, Yang J, Ye T, Zhang G, Zhang L, Zhao G, Zhao J, Zhong WZ, Zhu Y, Hulsewé KWE, Vissers YLJ, de Loos ER, Jeong JY, Marulli G, Sandri A, Sziklavari Z, Vannucci J, Ampollini L, Ueda Y, Liu C, Bille A, Hamaji M, Aramini B, Inci I, Pompili C, Van Veer H, Fiorelli A, Sara R, Sarkaria IS, Davoli F, Kuroda H, Bölükbas S, Li XF, Huang L, Jiang T. Expert consensus on resection of chest wall tumors and chest wall reconstruction. Transl Lung Cancer Res 2021;10(11):4057-4083. doi: 10.21037/tlcr-21-935 